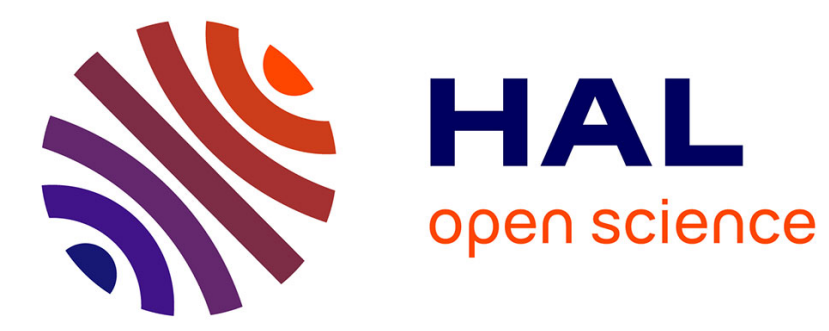

\title{
Spectroscopic analysis of accretion/ejection signatures in the Herbig Ae/Be stars HD 261941 and V590 Mon
}

\author{
T Moura, S. Alencar, A. Sousa, E. Alecian, Y. Lebreton
}

\section{To cite this version:}

T Moura, S. Alencar, A. Sousa, E. Alecian, Y. Lebreton. Spectroscopic analysis of accretion/ejection signatures in the Herbig Ae/Be stars HD 261941 and V590 Mon. Monthly Notices of the Royal Astronomical Society, 2020, 494 (3), pp.3512-3535. 10.1093/mnras/staa695 . hal-02523038

\section{HAL Id: hal-02523038 \\ https://hal.science/hal-02523038}

Submitted on 16 May 2020

HAL is a multi-disciplinary open access archive for the deposit and dissemination of scientific research documents, whether they are published or not. The documents may come from teaching and research institutions in France or abroad, or from public or private research centers.
L'archive ouverte pluridisciplinaire HAL, est destinée au dépôt et à la diffusion de documents scientifiques de niveau recherche, publiés ou non, émanant des établissements d'enseignement et de recherche français ou étrangers, des laboratoires publics ou privés. 


\title{
Spectroscopic analysis of accretion/ejection signatures in the Herbig Ae/Be stars HD 261941 and V590 Mon
}

\author{
T. Moura ${ }^{1 \star}$, S. H. P. Alencar ${ }^{1}$, A. P. Sousa ${ }^{1,2}$, E. Alecian ${ }^{2}$, Y. Lebreton ${ }^{3,4}$ \\ ${ }^{1}$ Universidade Federal de Minas Gerais, Departamento de Física, Av. Antônio Carlos 6627, 31270-901, Brazil \\ ${ }^{2}$ Univ. Grenoble Alpes, IPAG, F-38000 Grenoble, France \\ ${ }^{3}$ LESIA, Observatoire de Paris, PSL Research University, CNRS, Sorbonne Universités, UPMC Univ. Paris 06, \\ Univ. Paris Diderot, Sorbonne Paris Cité, 92195 Meudon Cedex, France \\ ${ }^{4}$ Univ Rennes, CNRS, IPR (Institut de Physique de Rennes) - UMR 6251, F-35000 Rennes, France
}

Accepted XXX. Received YYY; in original form ZZZ

\begin{abstract}
Herbig Ae/Be (HAeBe) stars are the intermediate-mass analogs of low-mass T Tauri stars. Both groups may present signs of accretion, outflow and IR excess related to the presence of circumstellar discs. Magnetospheric accretion models are generally used to describe accreting $\mathrm{T}$ Tauri stars, which are known to have magnetic fields strong enough to truncate their inner discs and form accretion funnels. Since few HAeBe stars have had magnetic fields detected, they may accrete through a different mechanism. Our goal is to analyse the morphology and variability of emission lines that are formed in the circumstellar environment of HAeBe stars and use them as tools to understand the physics of the accretion/ejection processes in these systems. We analyse high resolution (R 47000) UVES/ESO spectra of two HAeBe stars - HD 261941 (HAe) and V590 Mon (HBe) that are members of the young ( 3 Myr) NGC 2264 stellar cluster and present indications of sufficient circumstellar material for accretion and ejection processes to occur. We determine stellar parameters with synthetic spectra, and also analyse and classify circumstellar lines such as $\mathrm{H} \alpha, \mathrm{H} \beta, \mathrm{He} \mathrm{I} \lambda 5875.7$, according to their morphologies. We model the $\mathrm{H} \alpha$ mean line profile, using a hybrid MHD model that includes a stellar magnetosphere and a disc wind, and find signatures of magnetically driven outflow and accretion in HD 261941, while the $\mathrm{H} \alpha$ line of V590Mon seems to originate predominantly in a disc wind.
\end{abstract}

Key words: accretion, accretion discs - techniques: spectroscopic - stars: pre-main sequence - stars: Herbig Ae/Be

\section{INTRODUCTION}

Herbig Ae/Be (HAeBe) stars were first identified by Herbig (1960). They are optically visible Pre-Main-Sequence (PMS) stars with intermediate mass, from 2 to $8 \mathrm{M}_{\odot}$ (Herbig 1960; Finkenzeller \& Mundt 1984; The et al. 1994), analogous to the counterparts lowmass T Tauri stars (TTS) (Reiter et al. 2018). HAeBe systems commonly present circumstellar discs (e.g. Boehler et al. 2018, 2017; Klaassen et al. 2013) and large spectroscopic and photometric variability (Strom et al. 1972a) due to a highly dynamic circumstellar environment that can be used as a tool to understand the physics of accretion/ejection processes, which result from the star-disc interaction (Mendigutía et al. 2011a).

The star-disc interaction in accreting T Tauri stars, called Classical T Tauri stars (CTTS), is magnetically controlled, generating accretion flows and outflows (Bouvier et al. 2007). The magneto-

^ E-mail: tatiana@fisica.ufmg.br spheric accretion scenario is well established both observationally and theoretically for CTTS (e.g. Johns-Krull et al. 1999; Alencar et al. 2005). However, there are still many questions about how accretion proceeds in $\mathrm{HAeBe}$ systems. Some observational evidences indicate that Herbig Ae (HAe) stars may be similar to CTTS and present magnetospheric accretion, while Herbig Be (HBe) stars may accrete through a different mechanism (e.g. Vink et al. 2002; Cauley \& Johns-Krull 2014, 2015; Ababakr et al. 2015).

Muzerolle et al. (2004) investigated accretion in the Herbig Ae star UX Ori, modelling the observed emission lines with a magnetospheric accretion model. They showed that this type of model could describe overall the observed line profiles in UX Ori systems and that these systems present several accretion features, such as strong emission lines and redshifted absorption components in the Balmer lines, the $\mathrm{NaD}$ doublet and the $\mathrm{CaII}$ infrared triplet. Cauley \& Johns-Krull (2014) analysed the HeI $\lambda 10830$ line, which is very sensitive to both accretion and outflow signatures, in a sample of 56 $\mathrm{HAeBe}$ stars. They did not find a clear evidence of magnetospheric 
accretion in $\mathrm{HBe}$ stars and they found accretion signatures in HAe stars compatible with a smaller magnetosphere than typical CTTS. Cauley \& Johns-Krull (2015) looked for magnetospheric accretion features, like high velocity redshifted absorptions in optical line profiles, in a large sample of HAeBe stars. They confirmed the results found in Cauley \& Johns-Krull (2014) and concluded that the circumstellar environment of HAeBe stars could not be treated as a purely scaled analog of CTTS.

Mendigutía et al. (2011b) applied shock models, within the magnetospheric accretion scenario, to a sample of $38 \mathrm{HAeBe}$ stars. They derived accretion rates from UV Balmer excess and found a typical mean mass accretion rate of $\sim 10^{-7} \mathrm{M}_{\odot} \mathrm{yr}^{-1}$. However, the strong Balmer excesses and the line widths observed in the hottest Herbig Be stars of their sample could not be reproduced with magnetospheric accretion shock models, which led them to conclude that magnetospheric accretion may be present in HAe stars and that in HBe systems accretion is governed by some other mechanism. Fairlamb et al. (2015) analysed the spectra of $91 \mathrm{HAeBe}$ systems and found that $68 \%$ of the stars presented UV excess and $89 \%$ of them could have the mass accretion rate determined with a magnetospheric accretion model prescription. Despite these results, it is still unclear whether the magnetospheric accretion model applies to most HAeBe systems, since the detection rate of magnetic fields in these systems remains very low.

Through a high-resolution spectropolarimetric study, Alecian et al. (2013) detected magnetic fields in only $10 \%$ of the $70 \mathrm{HAeBe}$ stars of their sample. Wade et al. (2007) found that 8 to $12 \%$ of the $50 \mathrm{HAeBe}$ stars in their sample presented magnetic field strength up to $200 \mathrm{G}$. With a smaller sample of $21 \mathrm{HAeBe}$ stars and using spectropolarimetric observations, Hubrig et al. (2009) detected magnetic fields in 6 stars, the largest longitudinal magnetic field presenting $454 \mathrm{G}$. In contrast, the HAe star HD 101412 presents a very strong $3.5 \mathrm{kG}$ longitudinal magnetic field (Hubrig et al. 2013), but this is rather an exception among HAeBe systems. Overall, these results indicate that magnetic fields have been detected in only a small fraction of HAeBe stars. These magnetic fields appear to be stable over the years and to be mainly dipolar with polar strengths ranging from $\sim 300 \mathrm{G}$ to $\sim 3 \mathrm{kG}$. Such properties are fundamentally different from what is observed in T Tauri stars. In particular, when a $\mathrm{T}$ Tauri star presents a deep convective envelope, magnetic field detection is ubiquitous (Gregory et al. 2012). However, as soon as the convective envelope becomes less massive than about $2 \mathrm{M}_{\odot}$, magnetic fields are generally not detected anymore, and most of these stars have magnetic field strength limits of several hundred Gauss (Villebrun et al. 2019). While some of the non-detections could be due to stellar rotational velocities $(v \sin i)$ which are too large to allow the detection of typical $\mathrm{kG}$ magnetic fields, $v \sin i$ cannot be the only reason of non-detection in $90 \%$ of the stars which are mainly radiative. The absence of a deep convective envelope means that most of those stars cannot have surface magnetic fields stronger than several hundred Gauss.

The different magnetic properties, and especially the magnetic field strength distributions, between the T Tauri and HAeBe stars, must be addressed when invoking magnetospheric accretion onto $\mathrm{HAeBe}$ stars. It is assumed that magnetospheric accretion can happen when the magnetic pressure balances the ram pressure in the disc, which implies the disc to be truncated at a certain distance from the stellar surface. In the case of CTTS, kG fields and mass accretion rates of the order of $10^{-8} \mathrm{M}_{\odot} / \mathrm{yr}$ are typical and allow the disc to be truncated at several stellar radii from the stellar surface. According to Bessolaz et al. (2008), the truncation radius decreases when the magnetic strength decreases and the mass accretion rate increases. In the majority of HAeBe stars, magnetic fields are at least 2 times lower than in typical CTTS, while mass accretion rates appear to be between 1 to 10 times larger. This would reduce the truncation radius. However, the truncation radius increases with stellar radius. Herbig Ae/Be stars being between 2 to 4 times larger than $\mathrm{T}$ Tauri stars, even with weak fields, they can truncate the disc at several stellar radii. Therefore, according to the current observed properties of $\mathrm{HAeBe}$ stars, magnetospheric accretion remains a possible scenario.

Given all the uncertainties to interpret the accretion and outflow processes at work in HAeBe systems, an in depth analysis of a few HAeBe stars using modeling tools, in addition to observations, seems appropriate. In this work, we intend to provide a detailed analysis of the spectroscopic properties of two HAeBe stars belonging to the young cluster NGC 2264, V590 Mon and HD 261941, and discuss how we can relate the spectroscopic observations to the accretion/ejection processes in these systems.

V590 Mon (CSIMon-000392, Lk H $\alpha$ 25, Walker 90) is one of the most unusual stars belonging to the young cluster NGC 2264 (Breger 1974; Rydgren 1977; Pérez et al. 2008). Estimates of the spectral type of this star vary from A2 to B4 (e.g. Walker 1956; Warner et al. 1977; Sagar \& Joshi 1983) and Pérez et al. (2008) associated the different spectral types to temperature variability due to accretion episodes. The visual extinction of V590 Mon ranges from $\mathrm{A}_{v}=0.42$ (Liu et al. 2011) to $\mathrm{A}_{v}=5$ (Strom et al. 1972a). This anomalous extinction was attributed to the presence of grains of unusual sizes in its circumstellar environment (Rydgren 1977; Sitko et al. 1984), difficulting the determination of trustful deredenned colors. The spectral energy distribution of V590 Mon is unusual when compared with similar stars. Its SED increases by almost a factor of ten between 1 and $20 \mu \mathrm{m}$, while young stars of the same spectral type and age normally show a decrease in energy in this spectral region. To explain this feature, Strom et al. (1972b); Sitko et al. (1984); Rydgren \& Vrba (1987) suggested that the circumstellar disc around this star is seen nearly edge-on. Hillenbrand et al. (1992) analysed the spectral energy distributions of a sample of Herbig Ae/Be stars and classified V590 Mon in their Group II, that shows flat or rising infrared spectra. This group includes young stars surrounded by dusty envelopes that intercept a significant solid angle in our line of sight. Variability at both optical and infrared wavelengths were reported by several authors (e.g. Walker 1956; Poveda 1965; Breger 1972; Bhatt \& Sagar 1992) and some of them suggested a relationship between the observed variability and a variable circumstellar shell. Bhatt \& Sagar (1992), however, suggested that a variation in the mass accretion rate could be responsible for the observed photometric variability. V590 Mon was considered a binary candidate for the first time by Thomas et al. (2007) with 5007 milli-arcsecond of separation and Wheelwright et al. (2010) confirmed the binary nature of this system.

Our second selected object, HD 261941 (CSIMon-000631, Walker 165) is also a member of the young cluster NGC 2264 (Vasilevskis et al. 1965), and is less documented in the literature than V590 Mon. Considered by most authors as a non-variable star (Walker 1956; Breger 1972; Warner et al. 1977; Zwintz et al. 2009), it has spectral type A5/A2 (e.g. McNamara 1976; Rydgren 1977; Young 1978; Paunzen et al. 2002; Clarke et al. 2005). HD 261941 shows a strong infrared excess (Strom et al. 1972a) and was classified by Mendoza V. \& Gomez (1980) as a variable radial velocity star. Its light curves obtained with the CoRoT and Spitzer satellites were classified as aperiodic and irregular by Cody et al. (2014).

The paper is organised as follows. In Section 2 we describe the analysed data and in Section 3 we present the evolutionary stage of 
our targets. In Section 4 we describe our targets selection and in Section 5 we show the physical parameters found for our two selected objects. Our spectroscopic analysis is described in Section 6 and the morphological classification of the main line profiles is discussed in Section 7. To complement our spectroscopic analysis, $\mathrm{H} \alpha$ profile models are compared to our data in Section 8. Discussions and conclusions of this work are presented in Section 9.

\section{OBSERVATIONS}

As part of the Coordinated Synoptic Investigation of NGC 2264 (CSI 2264) campaign (Cody et al. 2014), spectroscopic observations of T Tauri and Herbig Ae/Be systems were carried out during 21 nights from December 2011 to February 2012 and 13 nights from December 2013 to January 2014. In both periods, the data were obtained with the Very Large Telescope (VLT/ESO) at Cerro Paranal, Chile, equipped with the high-resolution $(\mathrm{R} \sim 47000)$ spectrograph Ultraviolet and Visual Echelle Spectrograph (UVES). UVES provides spectra covering the wavelength range of 4800$6800 \AA$, split in the lower, 4800-5800 , and upper, 5800-6800 , arms. In total 15 targets were observed with UVES, including HAeBe systems and CTTS members of the young cluster NGC 2264.

The data reduction was done using the Reflex program, provided by ESO, and the UVES reduction pipelines. Bias subtraction, flat field correction, and wavelength calibration were performed. For spectra processing, IDL routines were used.

In addition to the VLT spectroscopic observations, we analysed optical photometry obtained with the CoRoT satellite in two distinct epochs: 23 days in March 2008 and 40 days from December 2011 to January 2012, covering wavelengths $\sim 3700-10000 \AA$, and previously analysed as part of the CSI 2264 campaign (Cody et al. 2013).

To construct the HR diagram (Section 3) and Spectral Energy Distributions (SEDs) (Section 4) we used literature data. We obtained effective temperatures and stellar luminosities from Fairlamb et al. (2015); Flaccomio et al. (1999, 2006); Venuti et al. (2014); Zwintz et al. (2013, 2009); Neri et al. (1993); Lamm et al. (2004); Penston (1964). We obtained photometric data from the Wide-field Infrared Survey Explorer (WISE) at 3.4, 4.6, 12.0 and $22 \mu \mathrm{m}$ (Wright et al. 2010), IRAC and MIPs data from Sung et al. (2009), JHK fluxes from 2MASS and optical (UBVRI) data from Sung et al. (2008).

\section{THE EVOLUTIONARY STAGE OF OUR SAMPLE}

We used a grid of evolutionary tracks of solar abundance, computed with the CESTAM code (Morel \& Lebreton 2008; Marques et al. 2013) to produce the HR diagram of Fig. 1. Details on the grid calculation can be found in Villebrun et al. (2019). As can be seen in Fig. 1, stars with masses larger than $1.5 \mathrm{M}_{\odot}$ present considerable changes in their internal structure as they evolve in the PMS, starting fully convective (orange region), developing a radiative core (green region), later becoming totally radiative (blue region), and near the Main Sequence, the core becomes convective (pink region). These structural changes have strong influence in the capacity to generate and sustain a stable magnetic field and they may also affect the magnetic field complexity, variability, and strength (Gregory et al. 2012; Villebrun et al. 2019). The existence of an initial

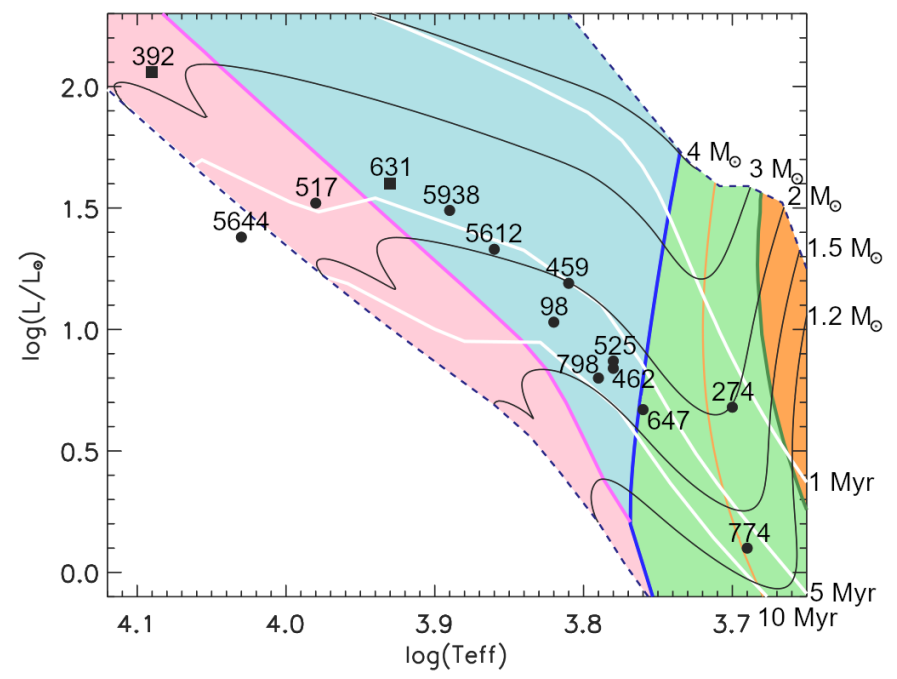

Figure 1. The HR diagram shows the distribution of 14 young stars observed with UVES as part of the CSI 2264 campaign (Cody et al. 2014). The evolutionary tracks were computed with the CESTAM code (Morel \& Lebreton 2008; Marques et al. 2013). The white lines correspond to the 1,5, $10 \mathrm{Myr}$ isochrones, from top to bottom. The black solid lines are the PMS evolutionary tracks for $1.2,1.5,2,3$, and $4 \mathrm{M}_{\odot}$, as labelled. The ZAMS (bottom) and the birthline (top) are represented by black dashed lines. The numbers above each star are their CSIMon identifications. The filled squares correspond to the analysed stars V590 Mon (star 392) and HD 261941 (star 631). In the orange region, stars are fully convective, in the green region stars present a radiative core and a convective envelope, the blue region corresponds to fully radiative systems and the pink region contains stars with a convective core and a radiative envelope.

phase with a convective envelope region in intermediate-mass systems may be responsible for the magnetic fields observed in a few of those stars (Alecian et al. 2017).

\section{SELECTION OF OBJECTS}

To investigate accretion and ejection processes, it was necessary to determine beforehand which systems had sufficient circumstellar material for these processes to occur. We analysed Spectral Energy Distributions (SEDs) of our sample of stars and selected two systems, V590 Mon (HBe) and HD 261941 (HAe), with substantial infrared emission excess, a characteristic feature of the presence of a circumstellar disc. The two selected systems differ in spectral type and also in terms of internal structure, as can be seen in Fig. 1. While HD 261941 has a totally radiative interior, V590 Mon presents a convective core and a radiative envelope. However, as the stellar features analysed in this work come from the stellar surface and beyond, a different structure in the core may not be relevant.

\subsection{SED model}

We modeled the SEDs of our selected sources, built with the photometric data described in Section 2. We used the Python-based fitting code sedfitter ${ }^{1}$ (Robitaille 2017) based on Hyperion, an

\footnotetext{
1 We used version v1.0 of the code, available to download at https://doi.org/10.5281/zenodo.235786.
} 
open-source parallelized three-dimensional dust continuum radiative transfer code by Robitaille (2011). The code is composed of modular sets ${ }^{2}$ with components that can include a stellar photosphere, a disc, an envelope, and ambipolar cavities. We used the five following sets of models: Model 1, which is composed of only a stellar photosphere, model 2 that includes a stellar photosphere and a passive disc, model 3, composed of a stellar photosphere, a passive disc, and an inner disc hole, model 4, which consists of a stellar photosphere, a passive disc and a dust envelope and model 5 that has a stellar photosphere, a passive disc, a dust envelope and an inner disc hole. The inner disc radius $\left(\mathrm{R}_{i n}\right)$ is set to the dust sublimation radius $\left(\mathbf{R}_{\text {sub }}\right)$ in discs without an inner hole (models 2 and 4) or varied from 1 to $1000 R_{\text {sub }}$ to achieve the best fit in the models with an inner disc hole. The input parameters of the Hyperion sedfitter are a range of interstellar redenning and distance from the sources to the Sun, and an estimate of the aperture used during the data reduction process. We used the parallax from the Gaia mission second release (Gaia Collaboration et al. 2016, 2018) to determine the individual distance of each system through Bayesian methods, following Luri et al. (2018) and $\mathrm{A}_{v}$ values from the literature (Tab. 1). The five modular sets were fitted to the the SED of our targets and the best model for each system was selected comparing the stellar output parameters from the SED fit with previously determined stellar temperatures and radii, listed in Tab. 1.

Fig. 2 shows the best SED fit models for V590 Mon and HD 261941. Black dots correspond to the observed fluxes taken from the literature, and the lines are the model results. Black lines represent the total flux output from the sedfitter code and dashed colored lines (when present) represent the emission components that were computed with the Hyperion code. Blue lines correspond to the stellar photospheric contribution and red lines represent the circumstellar contribution. The two last plots of Fig. 2 correspond to the SED fit of V590 Mon, which presents substantial IR excess. The model 2 result (stellar photosphere + passive disc) converged to radii and effective temperature values that were very different from those available in the literature for the system (Fairlamb et al. 2015). We therefore decided to adopt model 5 (stellar photosphere + passive disc + envelope + inner disc hole) and obtained much more consistent values of stellar radius and effective temperature. The adopted SED model of V590 Mon shows that an extended circumstellar structure is present around the system and is further justified by the report of the presence of an envelope in this system (e.g. Herbig 1960; The et al. 1994; Valenti et al. 2000, 2003).

\section{PHYSICAL PARAMETERS}

\subsection{Synthetic Spectra}

To analyse the circumstellar environment of our selected HAeBe systems it is necessary to identify the circumstellar contribution to the observed emission line profiles. We therefore removed the photospheric contribution from the observed emission lines, using synthetic spectra.

To construct the synthetic spectra, we used the line synthesis codes SME (Valenti \& Piskunov 1996) and BinMag4 (Kochukhov 2007) with the ATLAS9 atmosphere model (Kurucz 1993) and the spectral line data from the VALD3 database (Ryabchikova \&

2 All the modular sets of synthetic spectral energy distributions were downloaded from http://doi.org/10.5281/zenodo.166732, using version v1.1 of the models.
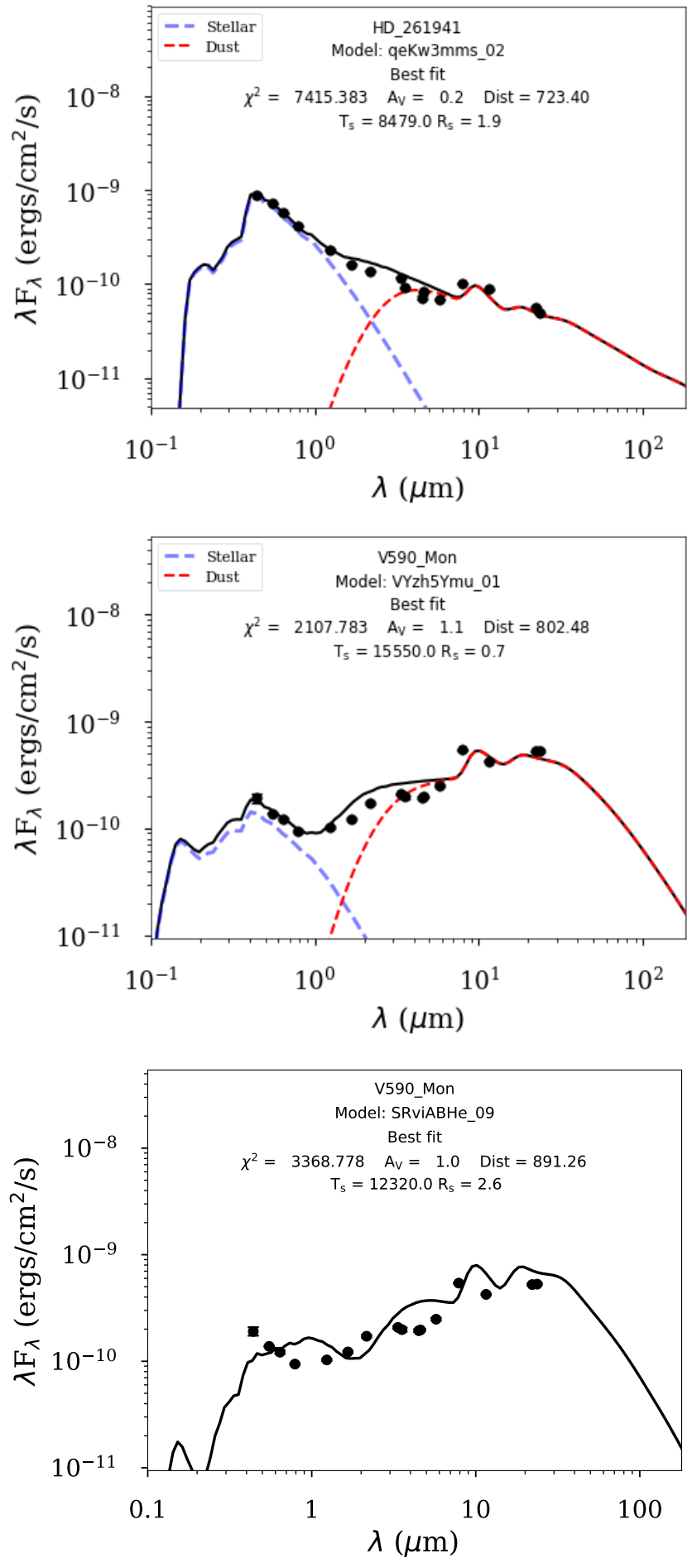

Figure 2. Spectral Energy Distributions of HD 261941 (top) and V590 Mon (middle and bottom). Dots correspond to the literature photometric data from 0.2 to $24 \mathrm{~m} \mu$ (see Sect. 2). The solid black line is the best fit of the Hyperion SED model and the dashed lines, when present, correspond to the stellar (blue) and dust emission (red) components (Robitaille 2011, 2017). The plots list the fitted values of the visual extinction $\left(\mathrm{A}_{v}\right)$ in mag, the source distance (Dist) in parsec, the star's effective temperature $\left(\mathrm{T}_{s}\right)$ in Kelvin, and the stellar radius $\left(\mathrm{R}_{s}\right)$ in solar radii. The top and middle panels correspond to models with an inner disc radius equal to the dust sublimation radius (model 2), while in the bottom panel V590 Mon was modeled with an inner disc radius larger than the dust sublimation radius $\left(\mathrm{R}_{i n}=4.32 \mathrm{R}_{s u b}\right.$, model 5), converging to $\mathrm{T}_{s}$ and $\mathrm{R}_{s}$ values that agree with previous determinations. 
Pakhomov 2015). We assumed solar abundance, a microturbulence velocity of $\mathrm{v}_{\text {mic }}=2 \mathrm{~km} \mathrm{~s}^{-1}$ and a macroturbulence velocity of $\mathrm{v}_{\text {mac }}=$ $5 \mathrm{~km} \mathrm{~s}^{-1}$ (Dunkin et al. 1997). We calculated synthetic spectra with the resolution of the UVES spectrograph $(\mathrm{R} \sim 47000)$.

For each star, rotationally broadened synthetic spectra were calculated using the SME and BinMag4 codes and compared through a $\chi^{2}$ minimization with the mean observed spectra, computed including all the observed nights. The best synthetic spectra were obtained with the BinMag4 code and Tab. 1 shows the best set of parameters we obtained for each system.

V590 Mon does not present many photospheric lines in our observed spectra. To guide the calculation of the synthetic spectrum we used the parameters obtained by Fairlamb et al. (2015). They analysed X-shooter echelle spectra that cover a large wavelength range from 3000 to $23000 \AA$ and compared the wings of the observed Balmer lines with a grid of Kurucz-Castelli models (Kurucz 1993; Castelli \& Kurucz 2004; Munari et al. 2005). They determined for V590 Mon $\mathrm{T}_{e f f}=12500 \pm 1000 \mathrm{~K}$ and $\log g=4.20$ \pm 0.30 . Within these values, we calculated synthetic models with $250 \mathrm{~K}$ and 0.5 dex steps in $\mathrm{T}_{\text {eff }}$ and $\log g$, respectively, and found that the best fit to our data corresponded to $\mathrm{T}_{e f f}=(12500 \pm 1500) \mathrm{K}$ and $\log g=4.5 \pm 0.5$. The same fitting procedure was applied to HD 261941 , leading to $\mathrm{T}_{e f f}=(8500 \pm 200) \mathrm{K}$ and $\log g=3.5 \pm 0.5$. The effective temperatures indicate that V590 Mon is a HBe star and HD 261941 is a HAe star. Both stars are fast rotators with $v \sin i$ $\sim 150 \mathrm{~km} \mathrm{~s}^{-1}$ and $\sim 120 \mathrm{~km} \mathrm{~s}^{-1}$ for V590 Mon and HD 261941, respectively.

After constructing the synthetic spectrum of each star, we subtracted the photospheric contribution from the observed spectra. The resulting spectra, shown in Figs. 3 and 4 correspond only to the circumstellar contribution and are related to the accretion/ejection processes at work in the star-disc systems.

\subsection{Mass Accretion Rate}

Mass accretion rate $\left(\dot{M}_{a c c}\right)$ is an important parameter to describe the evolution of the young star-disc system, since a significant fraction of the stellar mass is acquired from the disc during the PMS phase (Mendigutía et al. 2012). The knowledge of this parameter helps us understand the dynamics in the circumstellar environment, and its determination is of fundamental importance to calculations of the theoretical $\mathrm{H} \alpha$ emission line profiles (Section 8).

Emission lines, such as $\mathrm{H} \alpha$, carry accretion signatures and their luminosities are associated with the accretion luminosity (Muzerolle et al. 1998; Mendigutía et al. 2011b). We applied the relation determined by Fairlamb et al. (2017)

$\log \left(\frac{L_{a c c}}{L_{\odot}}\right)=2.09( \pm 0.06)+1.00( \pm 0.05) \times \log \left(\frac{L_{H \alpha}}{L_{\odot}}\right)$,

to calculate the accretion luminosity of our targets.

The $\mathrm{H} \alpha$ luminosity $\left(\mathrm{L}_{H \alpha}\right)$ was obtained with the relation between flux and line luminosity $\left(\mathrm{L}_{H \alpha}=4 \pi \mathrm{d}^{2} \mathrm{~F}_{H \alpha}\right)$ and the $\mathrm{H} \alpha$ line flux $\left(\mathrm{F}_{H \alpha}\right)$ was computed from the $\mathrm{H} \alpha$ equivalent width $\left(\mathrm{EW}_{H \alpha}\right)$, using the relation

$F_{H \alpha}=E W_{H \alpha} F_{c}(H \alpha)$,

where $F_{c}(H \alpha)$ is the continuum flux at the $\mathrm{H} \alpha$ line wavelength calculated from the CoRoT photometry. For HD 261941 we had simultaneaous $\mathrm{H} \alpha$ and CoRoT observations and we used the CoRoT magnitude of the star at the time of the spectroscopic observations to compute the continuum flux. For V590 Mon, the spectroscopic and photometric data were taken at different epochs and we used the mean CoRoT magnitude of the star to estimate the continuum flux near $\mathrm{H} \alpha$. The continuum flux near $\mathrm{H} \alpha$ is given by $F_{c}(H \alpha)=(v / \lambda) F_{0} \times 10^{\left[-0.4\left(\mathrm{mag}-A_{v}\right)\right]}$, where $v$ and $\lambda$ represent the central frequency and wavelength of the $\mathrm{R}$ band, $F_{0}$ is the zero point flux in the $\mathrm{R}$ filter $\left(F_{0}=2971.4 \mathrm{Jy}\right)$ and $m a g$ is the stellar magnitude measured with the CoRoT observations. The interstellar extinction $\left(A_{v} \sim 0.4\right)$ was obtained by Rebull et al. (2002) for HD 261941. For V590 Mon we adopted the value found by Fairlamb et al. (2015) $\left(A_{v}=1.03_{-0.05}^{+0.04}\right)$

With the accretion luminosity, calculated using the average $\mathrm{H} \alpha$ line flux from all the observed nights, described by Eq. 1, we can obtain the mass accretion rate, following Gullbring et al. (1998)

$\dot{M}_{H \alpha}=\frac{L_{a c c} R_{*}}{G M_{*}\left(1-\frac{R_{*}}{R_{T}}\right)}$

in which $M_{*}$ is the stellar mass, $R_{*}$ is the stellar radius and $R_{T}$ is the disc truncation radius.

Young stellar objects that harbour a magnetic field will truncate their inner discs at a distance from the star, where the ram pressure, exerted by material moving through the disc due to accretion process, equals the stellar magnetic field pressure. For accretion to occur, the truncation radius $\left(R_{T}\right)$ must be smaller than the corotation radius $\left(R_{c o r}\right)$. We calculated the corotation radius, according to the prescription of Mendigutía et al. (2011b) and used it as an upper limit to the truncation radius in our models.

Mendigutía et al. (2011b) calculated corotation radii of several intermediate mass stars, assuming a circumstellar disc in keplerian rotation and using the width of the $\mathrm{H} \alpha$ line at $10 \%$ of maximum intensity $\left(\left\langle W_{10}(H \alpha)\right\rangle\right)$. The corotation radius corresponds to the disc radius that presents the same angular velocity as the star:

$R_{c o r} \sim \frac{v_{g a s} R_{*}}{v_{*}}$

where $v_{\text {gas }}$ is the azimuthal velocity of the gas at corotation and $R_{*}$ and $v_{*}$ are the stellar radius and equatorial rotational velocity. Since $v_{*} \geq v \sin i$, and assuming that the width of the $\mathrm{H} \alpha$ circumstellar line represents the gas velocity somewhere between $R_{*}$ and $R_{c o r}$, $v_{\text {gas }} \leq\left\langle W_{10}(H \alpha)\right\rangle / 2$, they obtained that

$R_{\text {cor }} \leq \frac{\left\langle W_{10}(H \alpha)\right\rangle R_{*}}{2 v \sin i}$

Mendigutía et al. (2011b) obtained $\mathrm{R}_{\text {cor }}=\sim 1-5 \mathrm{R}_{*}$ for typical values of $\left\langle W_{10}(H \alpha)\right\rangle$ and vsin $i$ in their sample. For HD 261941, with vsin $i=(120 \pm 20) \mathrm{km} \mathrm{s}^{-1}$ and $\left\langle W_{10}(H \alpha)\right\rangle=(537 \pm 32) \mathrm{km} \mathrm{s}^{-1}$, we got $\mathrm{R}_{c o r}=\left(2.2_{-0.4}^{+0.6}\right) \mathrm{R}_{*}$. For V590 Mon, with $\operatorname{vin} i=(150 \pm$ $50) \mathrm{km} \mathrm{s}^{-1}$ and $\left\langle W_{10}(H \alpha)\right\rangle=(578 \pm 30) \mathrm{km} \mathrm{s}^{-1}$, we obtained $\mathrm{R}_{c o r}$ $=\left(1.9_{-0.5}^{+1.1}\right) \mathrm{R}_{*}$.

Using Eq. 3 and the values listed in Tab. 1, we calculated the mass accretion rates for each system, obtaining $\dot{M}_{a c c}=\left(3.4_{-1.3}^{+4.6}\right) \times$ $10^{-7} \mathrm{M}_{\odot} \mathrm{yr}^{-1}$ for V590 Mon and $\dot{M}_{a c c}=\left(2.0_{-0.7}^{+1.0}\right) \times 10^{-7} \mathrm{M}_{\odot}^{-1.3} \mathrm{yr}^{-1}$ for HD 261941.

\section{SPECTROSCOPIC VARIABILITY ANALYSIS}

We analysed the temporal variability of selected spectral lines, to infer the physical processes at work in the circumstellar environment of HAeBe systems. 
Table 1. Set of parameters of V590 Mon and HD 261941.

\begin{tabular}{|c|c|c|c|}
\hline Parameter & V590 Mon & HD 261941 & Ref \\
\hline $\mathrm{A}_{v}(\mathrm{mag})$ & $1.03_{-0.05}^{+0.04 a}$ & $0.40^{b}$ & $a, b$ \\
\hline $\mathrm{d}[\mathrm{pc}]$ & $826 \pm 64$ & $759 \pm 36$ & Gaia Collaboration et al. (2018) \\
\hline $\mathrm{M}_{*}\left[\mathrm{M}_{\odot}\right]$ & $3.2 \pm 0.5$ & $2.32_{-0.18}^{+0.20}$ & This Work \\
\hline $\mathrm{R}_{*}\left[\mathrm{R}_{\odot}\right]$ & $2.15 \pm 0.20$ & $2.85 \pm 0.50$ & This Work \\
\hline $\log L_{*}\left[L_{\odot}\right]$ & $2.06 \pm 0.37^{a}$ & $1.6 \pm 0.1^{c}$ & $a, c$ \\
\hline \multirow[t]{2}{*}{$\operatorname{Disc}^{d}$} & $\mathrm{E}$ & $\mathrm{Y}$ & This Work \\
\hline & & & BinMag4 code (This Work) \\
\hline$T_{e f f}[\mathrm{~K}]$ & $12500 \pm 1500$ & $8500 \pm 200$ & \\
\hline $\log g$ & $4.5 \pm 0.5$ & $3.5 \pm 0.5$ & \\
\hline$v_{\text {rad }}\left[\mathrm{km} \mathrm{s}^{-1}\right]$ & $40 \pm 10$ & $22 \pm 2$ & \\
\hline \multirow[t]{2}{*}{$v \sin i\left[\mathrm{~km} \mathrm{~s}^{-1}\right]$} & $150 \pm 50$ & $120 \pm 20$ & \\
\hline & & & $I D L$ routines (This Work) \\
\hline $\mathrm{L}_{a c c}\left[\mathrm{~L}_{\odot}\right]$ & $7.3_{-1.8}^{+2.4}$ & $2.6_{-0.7}^{+0.8}$ & \\
\hline $\mathrm{R}_{\text {cor }}\left[\mathrm{R}_{*}\right]$ & $1.9_{-0.1}^{+1.8}$ & $2.2_{-0.4}^{+0.6}$ & \\
\hline$\left\langle W_{10}(H \alpha)\right\rangle\left[\mathrm{km} \mathrm{s}^{-1}\right]$ & $578 \pm 30$ & $537 \pm 32$ & \\
\hline$\dot{M}_{a c c}\left[\mathrm{M}_{\odot} \mathrm{yr}^{-1}\right.$ & $\left(3.4_{-1.3}^{+4.6}\right) \times 10^{-7}$ & $\left(2.0_{-0.7}^{+1.0}\right) \times 10^{-7}$ & \\
\hline $\mathrm{P}$ [days] & $0.6_{-0.3}^{+0.9}$ & $1.2_{-0.5}^{+0.9}$ & \\
\hline $\mathrm{P}_{\max }[$ days] & $0.78^{-0.3}$ & 1.22 & \\
\hline $\mathrm{i}\left[{ }^{\circ}\right]$ & $\sim 51$ & $\sim 79$ & \\
\hline
\end{tabular}

${ }^{a}$ Fairlamb et al. (2015); ${ }^{b}$ Venuti et al. (2014); ${ }^{c}$ Penston (1964); ${ }^{d}$ Inner disc detected by the Hyperion SED fitting code (Y), inner disc + envelope (E) (see Fig. 2)

\subsection{Circumstellar Lines}

We analysed the circumstellar regions of HD 261941 and V590 Mon, investigating the variability of emission lines such as $\mathrm{H} \alpha$, $\mathrm{H} \beta, \mathrm{HeI} \lambda 5875.7, \mathrm{NaD} \lambda 5889.9, \mathrm{NaD} \lambda 5895.9,[\mathrm{OI}] \lambda 6300.3$ and [OI] $\lambda 6363.7$, which may present contributions from accretion and outflow regions.

\subsubsection{V590 Mon}

V590 Mon is a HBe star composed of a radiative envelope and a convective core, according to the CESTAM evolutionary model (Fig. 1). It presents considerable circumstellar material (see Fig. 2), part of which is expected to accrete onto the star. Circumstellar lines of V590 Mon indeed show indications of accretion and outflow, as discussed below.

The $\mathrm{H} \alpha$ line (Fig. 3a) is intense and wide, indicating that the circumstellar gas is accelerated at high speeds. But since V590 Mon is a fast rotator $\left(v \sin i \sim 150 \mathrm{~km} \mathrm{~s}^{-1}\right)$, we cannot directly attribute the large line width to acceleration due to magnetospheric accretion. Moreover, the $\mathrm{H} \alpha$ profiles do not show strong variability in morphology and intensity, which is a typical behavior in systems with magnetospheric accretion. However, differently from our observations, Pérez et al. (2008) showed H $\alpha$ profiles of V590 Mon with a variable morphology and a similar line broadening of \pm 400 $\mathrm{km} \mathrm{s}^{-1}$.

The $\mathrm{H} \beta$ line (Fig. 3b) of V590 Mon presented a double-peaked profile with more variability than $\mathrm{H} \alpha$ in morphology and intensity during our observations.

The HeI line $\lambda 5875.7$ (Fig. 3c) circumstellar component is mostly in absorption and slightly redshifted in our observations. Redshifted absorptions come from regions where the gas moves away from the observer, and can be produced by accretion infall.
The NaD presents a stable interstellar component in absorption that is slightly blueshifted. Superposed to the interstellar component, we see clear redshifted absorptions below the continuum up to velocities of $150 \mathrm{~km} \mathrm{~s}^{-1}$ (Fig. 3d), which could be related to accretion events.

This system also shows two forbidden oxygen lines in emission (Fig. 3e and f) typically associated with low density outflows. The [OI] $\lambda 6300$ and $\lambda 6363$ line profiles were fitted with a single gaussian and were found to be blueshifted by $-23.8 \mathrm{~km} \mathrm{~s}^{-1}$ and $-24.1 \mathrm{~km} \mathrm{~s}^{-1}$, respectively, confirming a probable outflow origin. McGinnis et al. (2018) analysed signatures of jets and winds in young stars, using the $[\mathrm{OI}] \lambda 6300$ emission line. One of their targets was V590 Mon and they decomposed the [OI] $\lambda 6300$ line with two blueshifted emission components, a strong one at $-22.8 \mathrm{~km} \mathrm{~s}^{-1}$ and a small intensity emission at $-47.6 \mathrm{~km} \mathrm{~s}^{-1}$. We recover the intense blueshifted component in [OI] $\lambda 6300$ and we do not see evidence of the small intensity and large blueshifted component in our data.

Blueshifted absorptions, produced by material moving towards the observer in a wind/outflow, were only observed in the $\mathrm{H} \beta$ line.

\subsubsection{HD 261941}

HD 261941 is a HAe fully radiative star, according to the CESTAM evolutionary model (Fig. 1). It shows variable emission lines with several features that are compatible with the magnetospheric accretion scenario, as discussed below, in agreement with the suggestion by Cauley \& Johns-Krull $(2014,2015)$ that HAes stars may have similar accretion characteristics as CTTS.

Fig. 4a shows the circumstellar component of the $\mathrm{H} \alpha$ line of HD 261941, which presented strong variability in intensity and morphology during our observations. The variability is more 


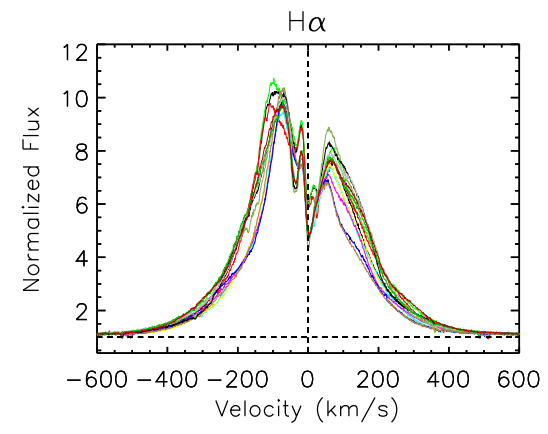

(a)

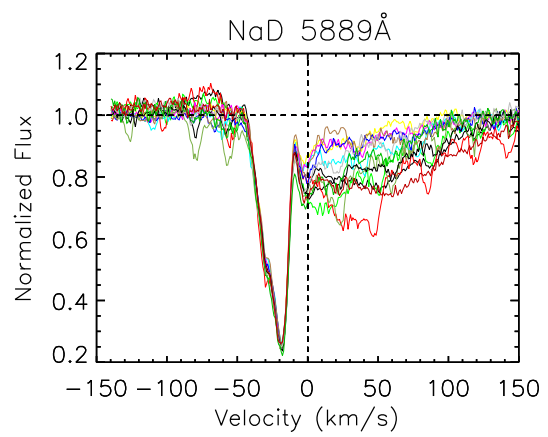

(d)

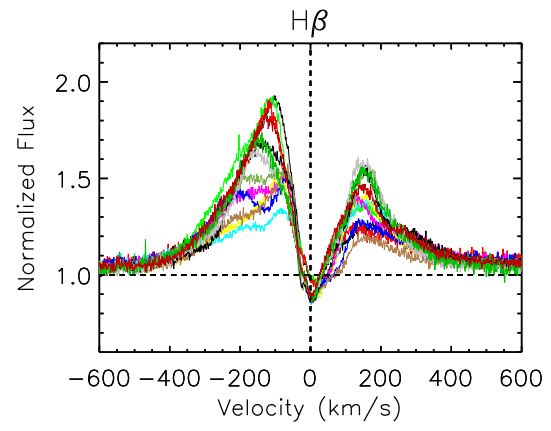

(b)

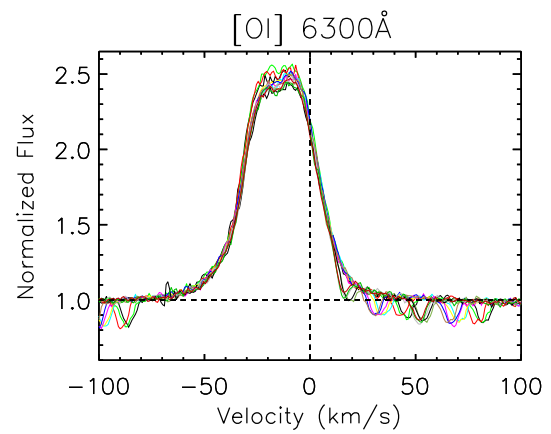

(e)

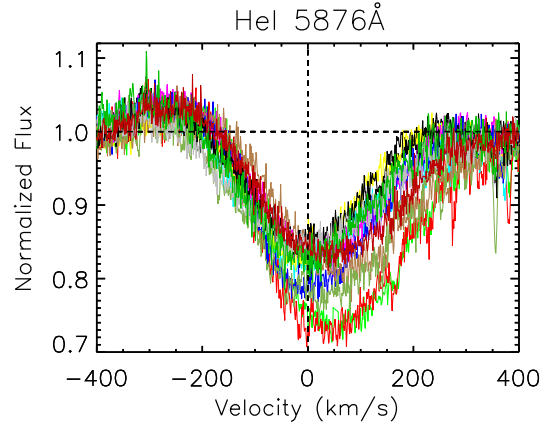

(c)

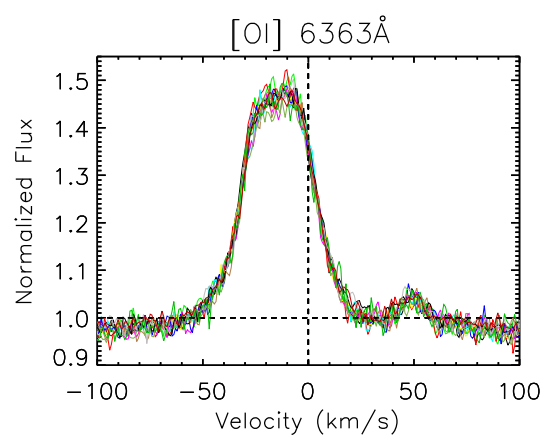

(f)

Figure 3. Circumstellar lines of V590 Mon: $\mathrm{H} \alpha$ (a), $\mathrm{H} \beta$ (b), He I $\lambda 5875.7$ (c), the NaD doublet (d) and the forbidden oxygen lines [OI] $\lambda 6300$ (e) and [OI] $\lambda 6363$ (f). The continuum corresponds to the horizontal dashed line and the stellar rest velocity is represented by a vertical dashed line. Each color represents one of the 13 observed UVES spectra.

prominent on the blue side of the line, with the presence of a blueshifted absorption component that sometimes goes below the continuum, indicating a possible wind origin. This line also has a large width, indicating the presence of gas at high speeds, but like V590 Mon, the star is rotating fast $\left(v \sin i \sim 120 \mathrm{~km} \mathrm{~s}^{-1}\right)$ and gas at high velocities cannot be directly related to magnetospheric accretion. The $\mathrm{H} \alpha$ emission line may be associated with winds (Cabrit et al. 1990) or a combination of magnetospheric accretion and winds (Kurosawa et al. 2006) in CTTS and HAeBe systems. Muzerolle et al. (2004), for example, were able to reproduce the observed $\mathrm{H} \alpha$ emission line of the HAe system UX Ori with the magnetospheric accretion model of Muzerolle et al. (2001).

The $\mathrm{H} \beta$ line (Fig. 4b) presents strong variability and blue and redshifted absorptions that go below the continuum, which may come from a wind and magnetospheric accretion funnels, respectively. The HeI line $\lambda 5875.7$ (Fig. 4c) is seen mostly in redshifted absorption in all the observed profiles. The $\mathrm{NaD}$ in this system is probably from the interstellar medium, presenting two very stable blueshifted absorption components.

\subsection{Correlation matrices}

The circumstellar lines discussed in the previous section show variability in different line regions. We can analyse how the variabilities are correlated across a line and between different lines, using correlation matrices.

Correlation matrices correspond to 2D plots of the linear cor- relation coefficient $\left(r_{i j}\right)$ between the variations of profile intensities in two different velocity bins $i$ and $j$.

$r_{i j}=\frac{\sum_{m=1}^{N}\left(S_{m i}-\bar{S}_{i}\right)\left(S_{m j}-\bar{S}_{j}\right)}{\sum_{m=1}^{N}\left(S_{m i}-\bar{S}_{i}\right)^{2}}$,

where $N$ is the total number of spectra (observed nights), $i$ and $j$ are indices for each velocity interval, $S_{m i}$ and $S_{m j}$ are the profile intensities of night $m$ in intervals $i$ and $j$, respectively, and $\bar{S}_{i}$ and $\bar{S}_{j}$ are the mean intensities of all nights for each interval.

When $i=j, r(i, j)=1$ and the matrices are symmetrical with respect to the main diagonal. $r_{i j}=1$ corresponds to a perfect correlation, $r_{i j}=0$ represents no correlation and $r_{i j}=-1$ corresponds to maximum anti-correlation. If some region of the profile is correlated to another, they are probably related to a commom physical phenomenon, such as accretion or outflow (Johns \& Basri 1995a; Oliveira et al. 2000; Alencar \& Batalha 2002).

\subsubsection{V590 Mon}

Figure 5 shows the correlation matrix corresponding to the 13 nights of observation of the $\mathrm{H} \alpha$ line. Except for the region around the stellar rest velocity, the entire line is correlated, which indicates that most of this line is formed in a single region of the system. Many authors have suggested that HBe systems, like V590 Mon, are not simple scaled versions of CTTS, and that there is no clear evidence that magnetospheric accretion is ongoing in these systems 


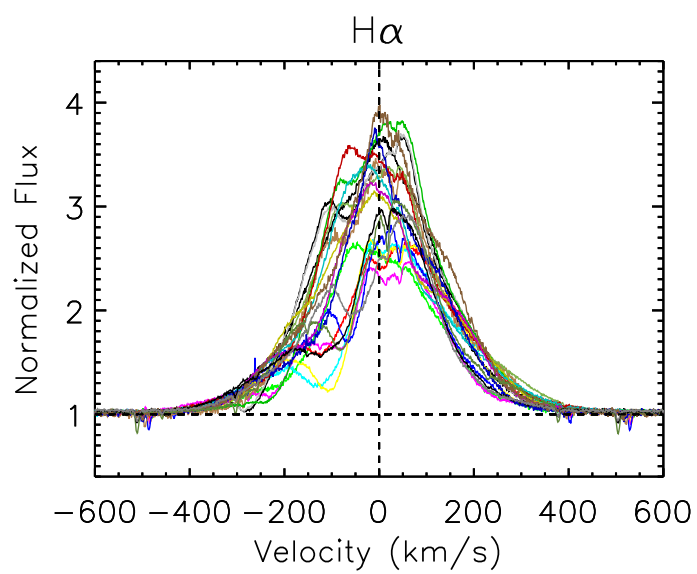

(a)

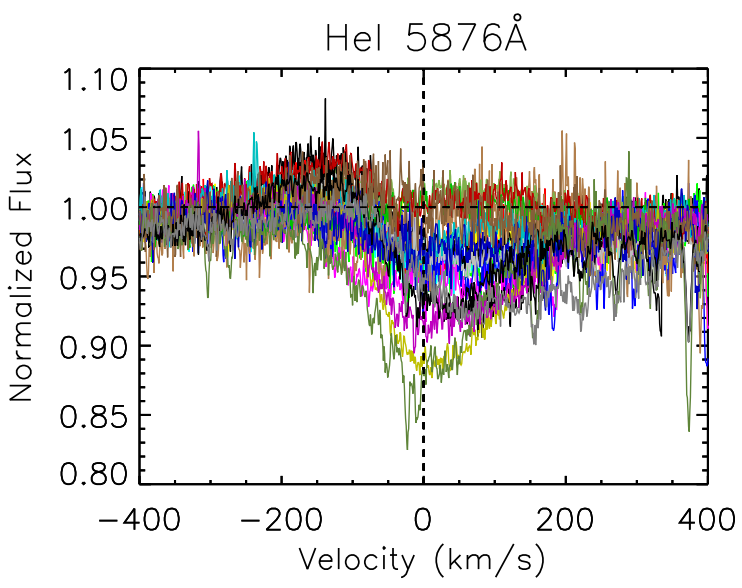

(c)

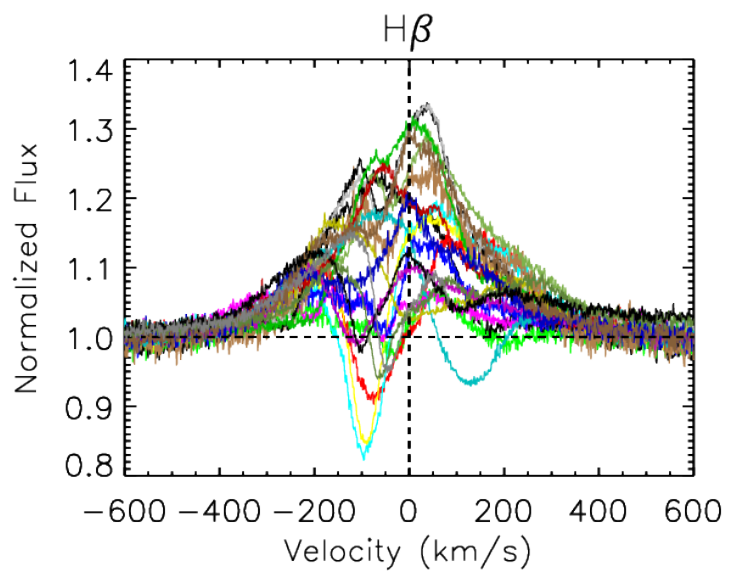

(b)

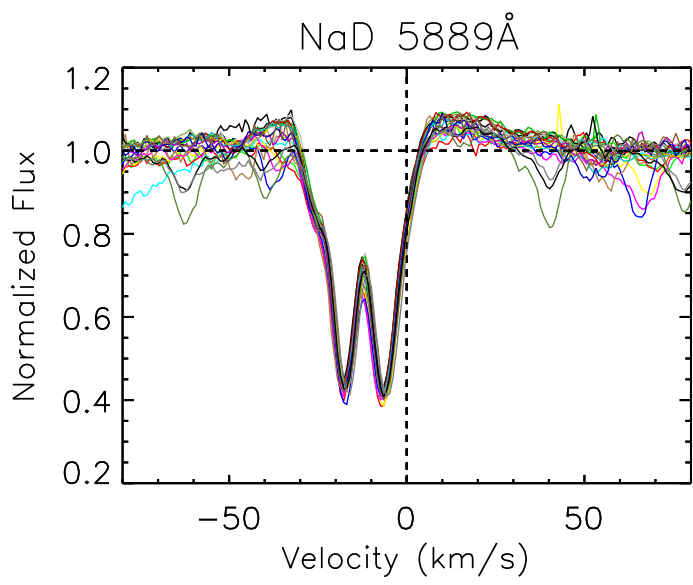

(d)

Figure 4. Selected circumstellar spectral lines of HD 261941: $\mathrm{H} \alpha$ (a), $\mathrm{H} \beta$ (b), He I $\lambda 5875.7$ (c) and the NaD doublet (d). The continuum corresponds to the horizontal dashed line and the stellar rest velocity is represented by a vertical dashed line. Each color represents one of the 21 UVES observed spectra.

(Vink et al. 2002; Cauley \& Johns-Krull 2014, 2015; Ababakr et al. 2015; Fairlamb et al. 2015; Schöller et al. 2016). The $\mathrm{H} \alpha$ emission line may therefore come mostly from a high density wind without a significant magnetospheric accretion contribution (Boehm \& Catala 1995).

Unlike $\mathrm{H} \alpha$, the $\mathrm{H} \beta$ line (Fig. 6) only presents correlations around the main diagonal, implying that the line comes from different regions that vary independently of each other. The red emission peak around $\mathrm{v}=200 \mathrm{~km} \mathrm{~s}^{-1}$ is furthermore anti-correlated with the blue side of the profile.

The HeI line $\lambda 5875.7$ (Fig. 7) shows strong correlation across the redshifted absorption, where most of the line variability is observed.

Analysis of correlation matrices between two distinct lines can indicate whether these lines are formed in the same region or not. In Figs. 8, 9 and 10 we present the correlation matrices between $\mathrm{H} \alpha, \mathrm{H} \beta$, and $\mathrm{HeI}$ 25875.7.

The correlation matrix between $\mathrm{H} \alpha$ and $\mathrm{H} \beta$ (Fig. 8) shows that the $\mathrm{H} \beta$ line correlates well with the entire $\mathrm{H} \alpha$ line, except for the $\mathrm{H} \beta$ profile beyond $150 \mathrm{~km} \mathrm{~s}^{-1}$. This indicates that the $\mathrm{H} \alpha$ line and most of the $\mathrm{H} \beta$ line come from a common formation region, like a wind, and that at least the variations of the red wing of $\mathrm{H} \beta$ are dominated by a different physical process, like accretion, for example. When modeling the $\mathrm{H} \alpha$ line in Section 8, this should be taken into account.

There is no correlation between the $\mathrm{He}$ I line and the $\mathrm{H} \beta$ and $\mathrm{H} \alpha$ lines (Figs. 9 and 10). If $\mathrm{H} \beta$ and $\mathrm{H} \alpha$ are mostly formed in a wind, this implies that the $\mathrm{He}$ I line comes mostly from a different circumstellar region, like the chromosphere or a boundary layer. The anti-correlations that appear in the matrices of $\mathrm{He} \mathrm{I}$ with $\mathrm{H} \beta$ and $\mathrm{H} \alpha$ are difficult to interpret either in a magnetospheric scenario or in a disc wind and boundary layer configuration.

\subsubsection{HD 261941}

Figure 11 presents the correlation matrix corresponding to the 21 nights of observation of the $\mathrm{H} \alpha$ line. There is mainly a small correlation around the diagonal, showing that different parts of the line tend to vary independently. The exceptions are the red $\left(0 \mathrm{~km} \mathrm{~s}^{-1}<\right.$ $\left.v<350 \mathrm{~km} \mathrm{~s}^{-1}\right)$ and blue $\left(-200 \mathrm{~km} \mathrm{~s}^{-1}<v<-400 \mathrm{~km} \mathrm{~s}^{-1}\right)$ regions, that show a strong anti-correlation with each other. This anti-correlation is somewhat reminiscent of the eggbeater scenario, 

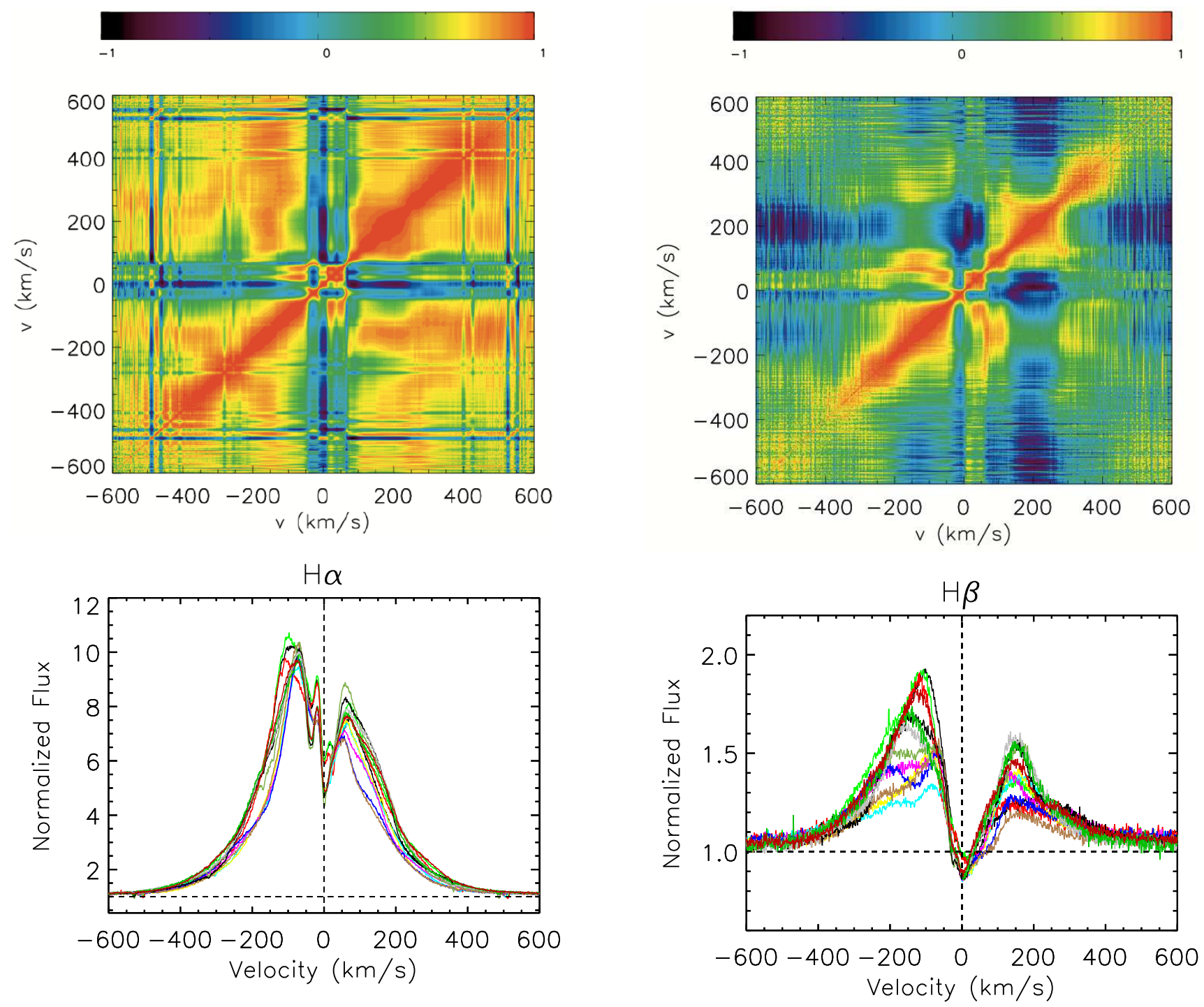

Figure 5. Auto-correlation matrix of the $\mathrm{H} \alpha$ line (left) and the corresponding emission line profiles of V590 Mon (right). The color bar above the matrix indicates the level of correlation.

based on a magnetospheric accretion model with an inclined dipole, proposed by Johns \& Basri (1995b) to explain the $\mathrm{H} \alpha$ line variability of the G2 classical T Tauri star SU Aur. In this model, accretion and outflow are favored at opposite rotation phases and as the system rotates we see successive accretion and outflow features in the line profiles.

Figure 12 shows the $\mathrm{H} \beta$ line and its correlation matrix. Like in $\mathrm{H} \alpha$, the most prominent feature is an anti-correlation between the red $\left(0 \mathrm{~km} \mathrm{~s}^{-1}<v<300 \mathrm{~km} \mathrm{~s}^{-1}\right)$ and blue $\left(-300 \mathrm{~km} \mathrm{~s}^{-1}<v<\right.$ $-200 \mathrm{~km} \mathrm{~s}^{-1}$ ) wings of the profiles.

The HeI line correlation matrix shows a strong correlation in the red side of the profile $\left(0 \mathrm{~km} \mathrm{~s}^{-1}<v<300 \mathrm{~km} \mathrm{~s}^{-1}\right)$, which corresponds to the redshifted absorption that dominates the profile variability. The variability of the redshifted absorption, which could be related to accretion, is not correlated with the changes in the blue side of the profile.

In Fig. 14 we analyse the variations of $\mathrm{H} \alpha$ with respect to $\mathrm{H} \beta$. The blue wings of both lines, where a highly variable blueshifetd absorption is present, are strongly correlated. This absorption is

Figure 6. Auto-correlation matrix of the $\mathrm{H} \beta$ line (left) and the corresponding emission line profiles of V590 Mon (right). The color bar above the matrix indicates the level of correlation.

typically seen in disc-wind models of CTTS (Kurosawa et al. 2006; Lima et al. 2010) and may therefore indicate a possible origin for the feature in HD 261941.

Figure 15 presents the correlation matrix of the $\mathrm{He} \mathrm{I}$ and $\mathrm{H} \alpha$ lines, which shows that the two lines are not well correlated. The only feature that presents some significance is a weak anticorrelation between the blue wing of $\mathrm{H} \alpha$ and the red side of $\mathrm{He}$ I that could be related to wind and accretion being favoured at opposite moments.

Figure 16 shows a correlation between the red wings of He I and $\mathrm{H} \beta$. This correlation points to a common origin, like an accretion funnel for example, for the redshift absorptions present in both lines.

\subsection{Period}

We investigated if the HAeBe systems showed any periodical variability in three distinct ways: (1) analysing the CoRoT light curves; 


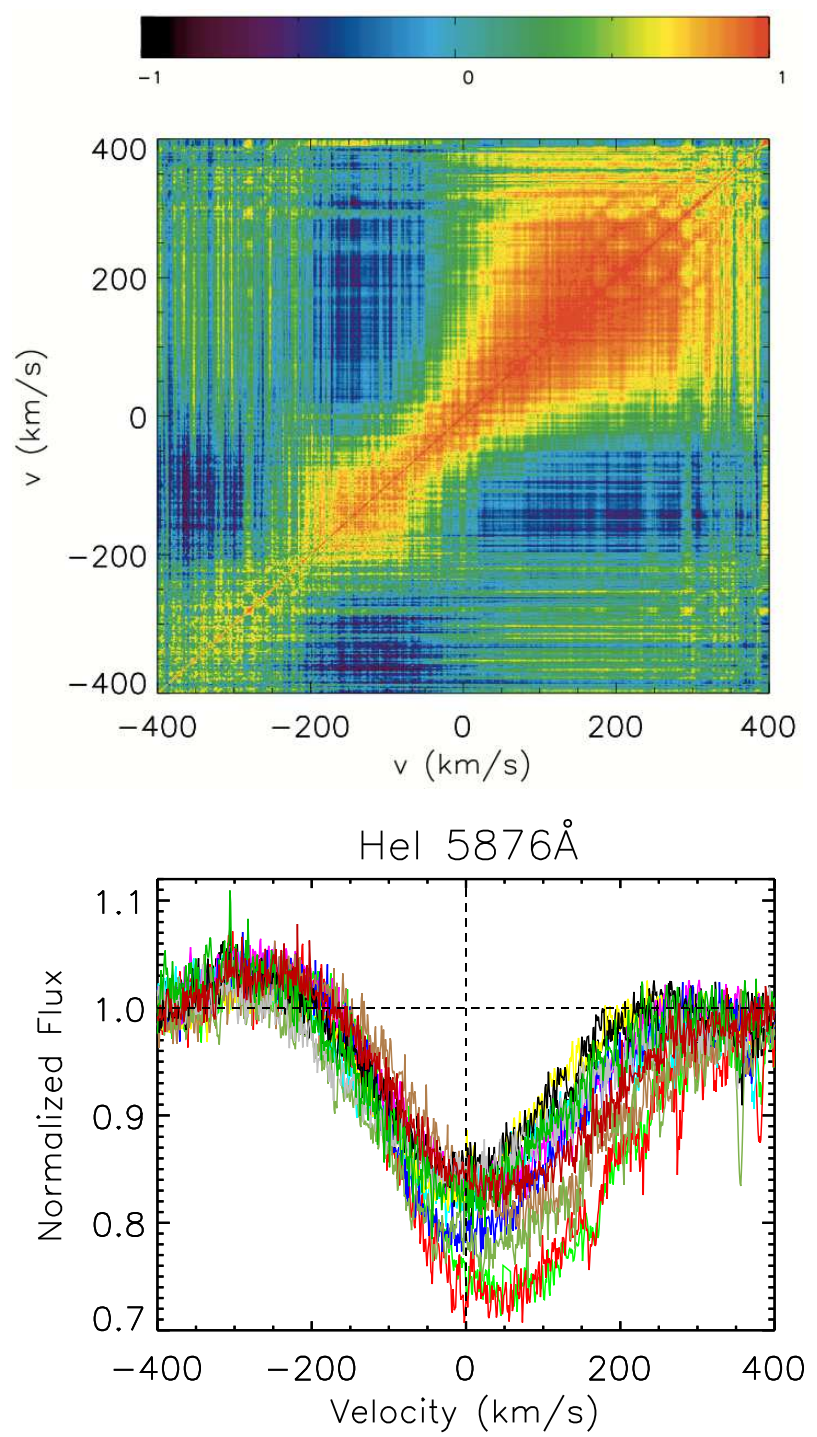

Figure 7. Auto-correlation matrix of the HeI $\lambda 5875.7$ line (left) and the corresponding observed line profiles of V590 Mon (right). The color bar above the matrix indicates the level of correlation.

(2) studying the circumstellar lines variability; (3) and relating the disc corotation radius to Kepler's third law and the $\mathrm{H} \alpha$ line width (Mendigutía et al. 2011b).

The best way to infer the stellar rotation period is through variation of photospheric features (such as spots or photospheric lines). Light curve variations due to the presence of spots are however often irregular and radial velocity variations of photospheric lines also depend on the presence of stable spots with a large temperature difference with respect to the photosphere, which are not expected in HAeBe systems.

Another possible way to obtain the stellar rotation period is through the variability of circumstellar lines, supposing that the line formation region rotates at the stellar rotation period. The circumstellar lines often present variabilities in their intensities and morphologies, but they may form in different regions that vary with different periods and, due to that, it is sometimes difficult to detect a single period in circumstellar lines of young stellar objects.

We looked for periodicities using the Scargle periodogram as modified by Horne \& Baliunas (1986) to handle non-equally spaced data. We calculated periodograms on the line profiles intensities in different velocity bins and present our results as $2 \mathrm{D}$ power plots that show which regions of the lines are periodic.

We also estimated the stellar rotation period following Mendigutía et al. (2011b) that related the width of the $\mathrm{H} \alpha$ line at $10 \%$ of maximum intensity to the corotation radius. The corotation radius is the radius at which the disc rotates at the stellar rotation period and corresponds to

$R_{\text {cor }}=\left(\frac{G M_{*} P^{2}}{4 \pi^{2}}\right)^{1 / 3}$.

We can then relate Eqs. 5 and 7

$\left(\frac{G M_{*} P^{2}}{4 \pi^{2}}\right)^{1 / 3} \leq \frac{\left\langle W_{10}(H \alpha)\right\rangle R_{*}}{2 v \sin i}$,

to obtain an upper limit of the stellar rotation period. Using the parameters from Tab. 1 together with Eq. 8, we calculated the upper value of the rotation period of V590 Mon and HD 261941, obtaining $\mathrm{P} \leq 0.6$ days and $\mathrm{P} \leq 1.2$ days, respectively. Using the relation $\operatorname{vsin} i=\frac{2 \pi R_{*} \sin i}{P}$, we can estimate the system inclination with respect to our line of sight, obtaining $\mathrm{i} \sim 51^{\circ}$ for V590 Mon and $\mathrm{i} \sim$ $79^{\circ}$ for HD 261941. In Section 8, while modeling the $\mathrm{H} \alpha$ emission profile, we will discuss again the system inclination with respect to our line of sight.

Taking into account our measured $v \sin i$ values and the stellar radius obtained from evolutionary models, we can also estimate the maximum stellar rotation period from

$P_{\text {max }} \leq \frac{2 \pi R_{*}}{v \sin i}$

obtaining $P_{\max }=0.78$ days for V590 Mon and $P_{\max }=1.22$ days for HD 261941, in agreement with the upper limits calculated in the previous paragraph.

\subsubsection{V590 Mon}

Fig. 17a shows the CoRoT light curve of V590 Mon from 2008, which presents a few days variability superposed to a long term variation. The periodogram analysis of the observed CoRoT light curve shows a periodic signature around 5 days with low power (Fig. 17b). To analyse only the short-term variability, we fitted the CoRoT light curve of V590 Mon with a 3rd degree polynomial and obtained the normalized light curve shown in Fig. 17d. The period search results in the normalized light curve confirmed the 5 dayperiod (Fig. 17e), with a higher power than the one obtained with the original light curve. The phase-folded light curves shown in Figs. $17 \mathrm{c}$ and $17 \mathrm{f}$ highlight the irregularity of the flux variations in the system. At the same time, the 5-day period cannot be associated with the stellar rotation period, that has a maximum value of 0.78 days, as calculated in the previous section. It can therefore be due to circumstellar material located farther away than the corotation radius in the disc or a wind, which could also explain the irregularity of the observed photometric variations.

The emission lines periodogram did not show a clear periodic signal, as can be seen in Fig. 18, with the exception of a period of about 4.2 days, from 0 to $400 \mathrm{~km} \mathrm{~s}^{-1}$, in the $\mathrm{HeI} \lambda 5875.7$ line that is however much longer than the maximum stellar rotation period of 0.78 days.

In the literature, periods of 4.47 days (Kearns \& Herbst 1998) and 27.18 days (Makidon et al. 2004) were associated with the photometric variability of V590 Mon. These periods are however much larger than the maximum value we determined, and are probably 


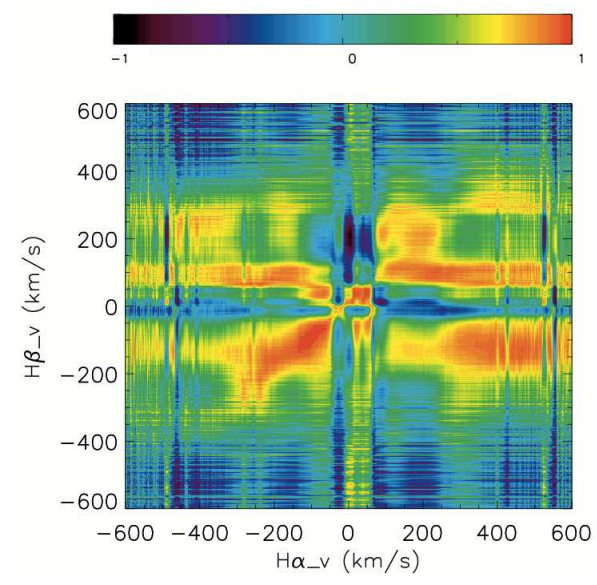

(a)

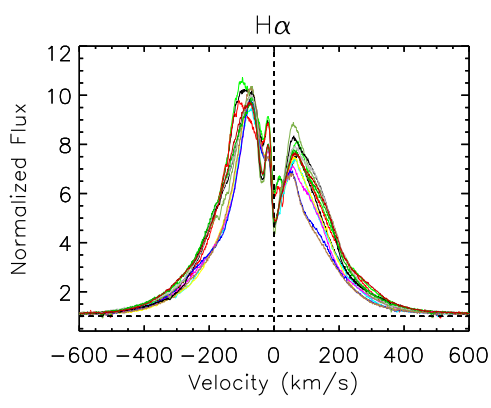

(b)

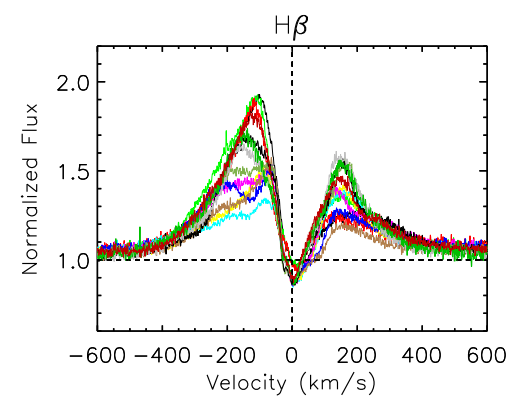

(c)

Figure 8. Correlation matrix (a) between $\mathrm{H} \alpha$ (b) and $\mathrm{H} \beta$ (c) of V590 Mon. The color bar above the matrix indicates the level of correlation.

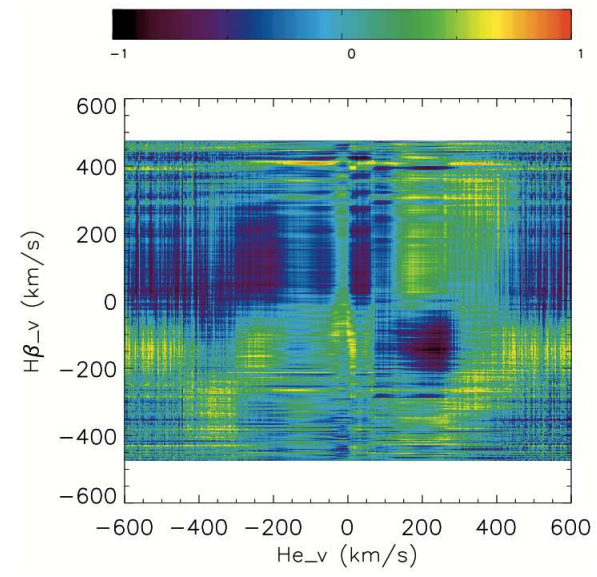

(a)

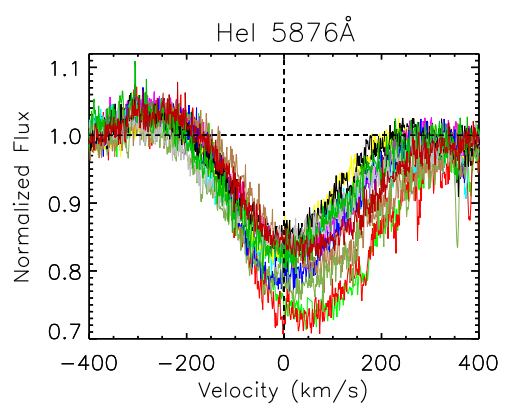

(b)

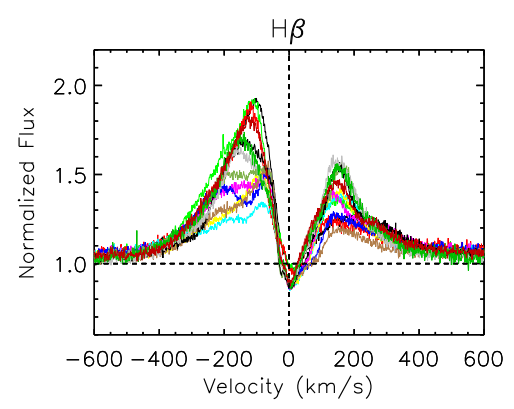

(c)

Figure 9. Correlation matrix (a) between HeI $\lambda 5875.7$ (b) and $\mathrm{H} \beta$ (c) of V590 Mon. The color bar above the matrix indicates the level of correlation.

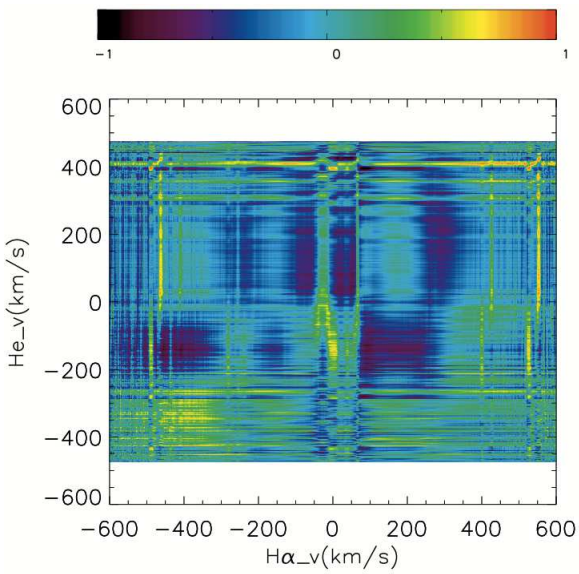

(a)

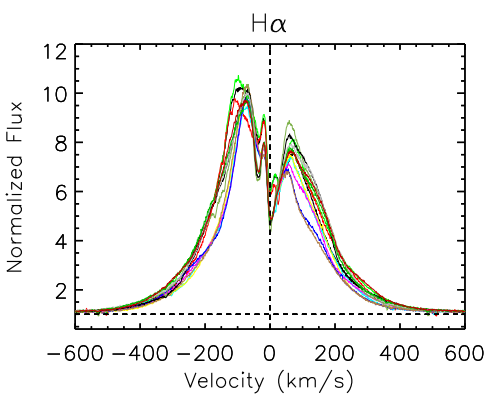

(b)

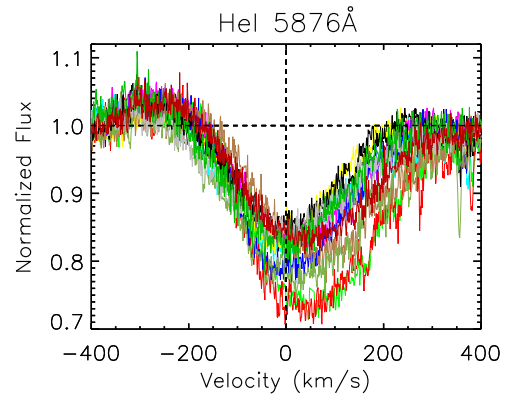

(c)

Figure 10. Correlation matrix (a) between $\mathrm{H} \alpha$ (b) and HeI $\lambda 5875.7$ (c) of V590 Mon. The color bar above the matrix indicates the level of correlation. 

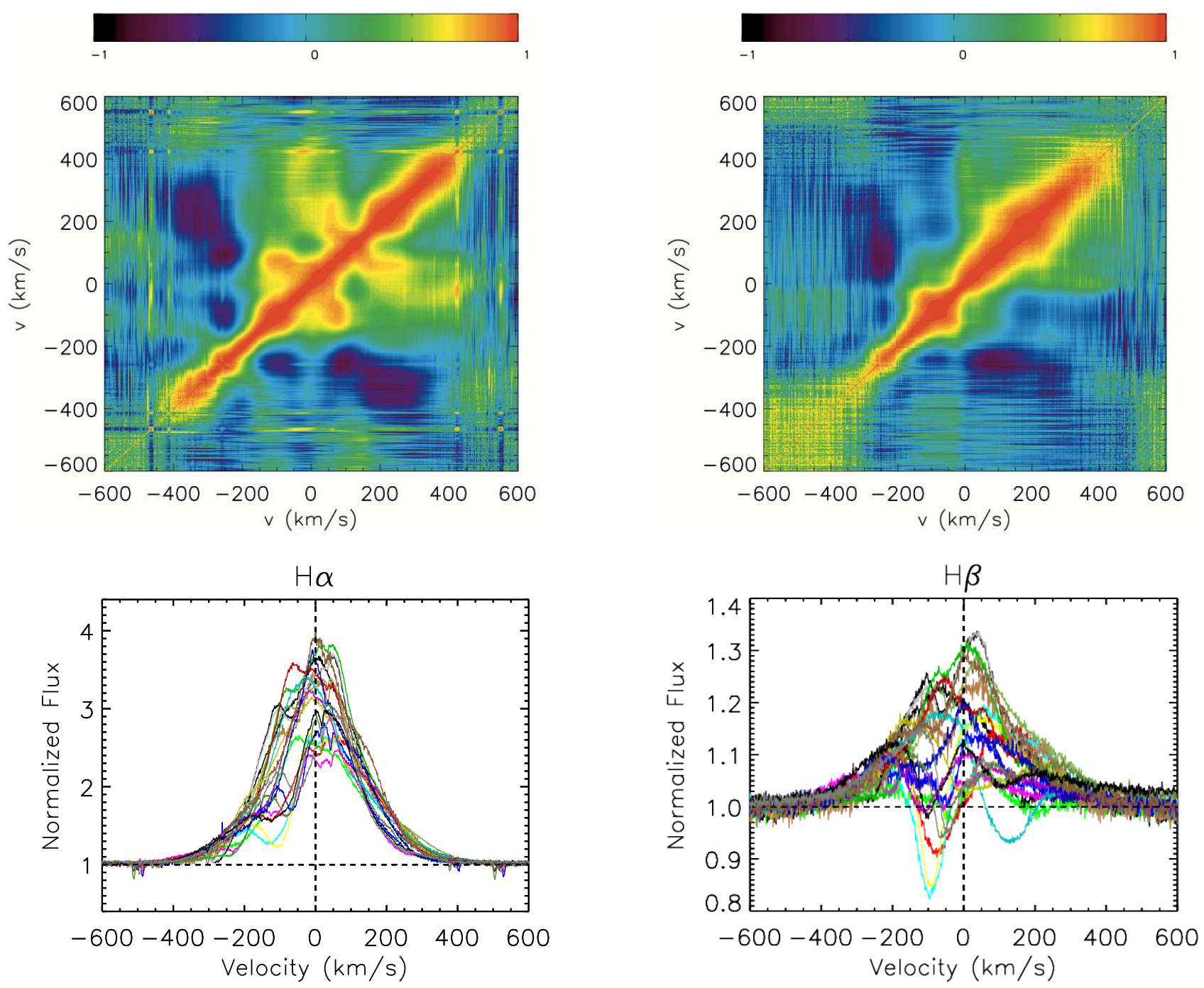

Figure 11. Auto-correlation matrix of the $\mathrm{H} \alpha$ line (top panel) and the corresponding line profiles of HD 261941 (bottom panel). The color bar above the matrix indicates the level of correlation.

due to circumstellar material located farther away than the corotation radius of the system.

\subsubsection{HD 261941}

Figs. 19a and 19d show the 2008 and 2011 CoRoT light curves of HD 261941, which are mostly constant in both epochs. A periodogram analysis finds a 6.7-day period in 2008 (19b) and a 8.0day period in 2011 (19e), however, the phase-folded light curves $(19 \mathrm{c}, \mathrm{f})$ do not present a periodic signal and we therefore did not consider the period detections as reliable.

We also looked for periodicities in the circumstellar lines of HD 261941. The H $\beta$ line shows a periodic signal around 12 days (Fig. 20) in the region that corresponds to the redshifted absorption component. $\mathrm{H} \alpha$ shows a period detection of 15 days in the blue wing, at the location of the highly variable blueshifted absorption. However, since our observations only cover 21 days, these remain as tentative period detections that should be investigated in future works covering a longer observation timespan.

Figure 12. Auto-correlation matrix of the $\mathrm{H} \beta$ line (top panel) and the corresponding line profiles (bottom panel) of HD 261941. The color bar above the matrix indicates the level of correlation.

\section{MORPHOLOGICAL CLASSIFICATION}

The morphological analysis of circumstellar lines in young systems provides a description of their circumstellar gas dynamics. In this section we will discuss all the circumstellar lines analysed by Cauley \& Johns-Krull (2015) in their sample of HAeBe stars and present in our observations of V590 Mon and HD 261941. We would like to study the incidence of accretion and outflow features in the spectra and compare our results with other observations of HAeBe systems.

We classified the circumstellar lines in groups, as proposed by Cauley \& Johns-Krull (2015) as: P-Cygni (PC), inverse P-Cygni (IPC), double-peaked emission (DP), single-peaked emission (E), absorption (A), and featureless (F). The evidence of absorption below the local continuum was classified as PC if the absorption occurs only in the blue side $\left(v<0 \mathrm{~km} \mathrm{~s}^{-1}\right)$ and as IPC if the absorption occurs only in the red side $\left(v>0 \mathrm{~km} \mathrm{~s}^{-1}\right)$. Profiles with any other absorption characteristic were classified simply as absorption profile (A).

Cauley \& Johns-Krull (2015) analysed a sample of 88 HAeBe 


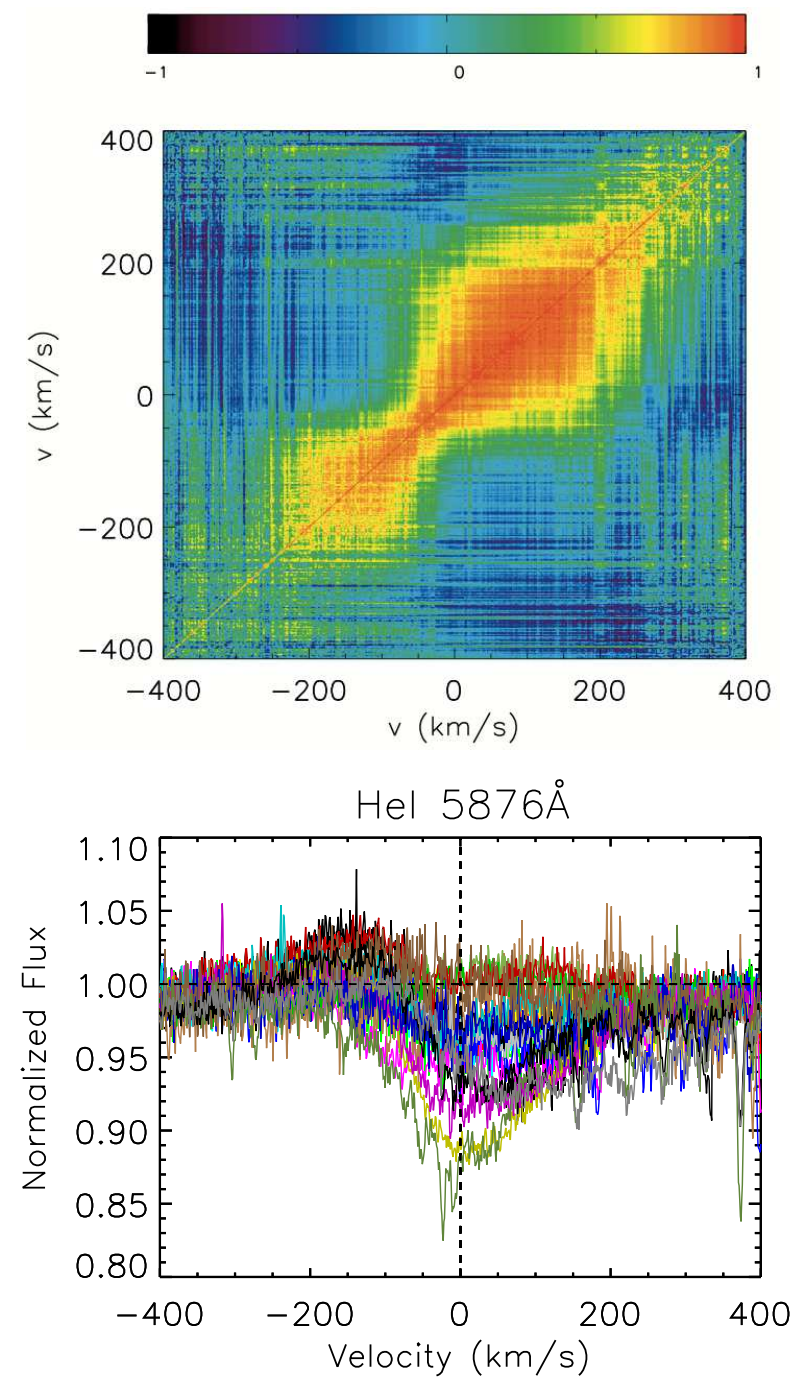

Figure 13. Auto-correlation matrix of the HeI 15875.7 line (top panel) and the corresponding line profiles (bottom panel) of HD 261941. The color bar above the matrix indicates the level of correlation.

stars (45 HAes and 33 HBes) and observed simultaneously red and blueshifted absorptions in only one star of the their sample, a Herbig Ae (A1 spectral type). In their sample, 19 stars presented redshifted absorptions, among them 15 were HAe stars and two of the HBes had spectral type B9. Assuming that redshifted absorptions below the continuum are an indication of high velocity accreting material as in CTTS, they concluded that HAe stars more often show evidence of magnetically controlled accretion than HBe systems. Evidence of outflows, such as blueshifted absorptions, are similar in HAes and HBes, suggesting that a wind is commonly present in both groups.

In the following sections we analyse the line profile morphology of our stars. V590 Mon presented emission lines with a stable profile morphology, and we only discuss the mean line profile of each line, while the circumstellar line profiles of HD 261941 varied a lot and it was necessary to analyse each observation separately.

\subsubsection{V590 Mon}

We analysed 17 circumstellar lines of V590 Mon. Three presented IPC profiles, 2 DP profiles, 4 only emissions, and 2 only absorptions. We did not find PC profiles and 6 lines were classified as featureless, as can be seen in Fig. 21. From these results, we found some evidence of accretion through the IPC profiles and no clear wind signature, due to a lack of blueshifted absorption components.

V590 Mon was also investigated by Cauley \& Johns-Krull (2015). We could then compare our morphological analysis with their results, as shown in Fig. 22. They analysed the morphology of 26 circumstellar lines of V590 Mon. They found 2 DP profiles, 4 emission profiles and 20 featureless lines and did not find any PC, IPC or absorption profiles in their spectra. Pérez et al. (2008) presented $\mathrm{H} \alpha$ and $\mathrm{H} \beta$ line profiles of V590 Mon which showed different morphological characteristics when compared to our observations. Their $\mathrm{H} \alpha$ observations showed inverse P-Cygni profiles and the $\mathrm{H} \beta$ line presented blueshifted and redshifted absorption components. According to them, the detection of the IPC in many spectral lines demonstrated the evidence of gas infall, leading them to suggest the presence of accretion flows in the system.

V590 Mon presents therefore line profiles with a variable morphology in different timecales. We analysed 13 nights of observations from 2013/2014, Cauley \& Johns-Krull (2015) analysed only one spectrum taken in 2013 and Pérez et al. (2008) investigated 7 spectra observed in 2002/2003. Different epochs of observation indicated different morphologies and variabilities, making it difficult to assign a single model to the circumstellar dynamics of this system.

\subsubsection{HD 261941}

The circumstellar lines of HD 261941 showed a large variability in morphology in our observations, as can be seen in Fig. A2. Due to that, we decided to analyse each profile separately, instead of using the mean line profiles. We show the results in Tab. 2, corresponding to 21 observations of 16 circumstellar lines.

The morphology of the $\mathrm{H} \alpha$ line changed from double-peaked emission to single-peaked emission, while $\mathrm{H} \beta$ presented various profile morphologies, including P-Cygni, inverse P-Cygni, doublepeaked emission, single-peaked emission and even featureless. The P-Cygni profiles observed in the $\mathrm{H} \beta$ line of HD 261941 show narrow absorptions that go below the continuum, while normally $\mathrm{P}$ Cygni profiles are associated with extended absorption components. The $\mathrm{H} \beta$ line presents both infall and outflow characteristic features, showing red and blueshifted absorptions below the continuum, as commonly observed in high inclination CTTS described by magnetospheric accretion models, such as AA Tau (K7, Bouvier et al. 1999; Esau et al. 2014) and SU Aur (G2, Johns \& Basri 1995b).

\section{H $\alpha$ MODEL}

The circumstellar contribution of the $\mathrm{H} \alpha$ line can come from accretion and outflow regions around young stellar objects. While the observed circumstellar lines of CTTS are well described by magnetospheric accretion models, there is not yet a consensus on the best model to describe the circumstellar environment of HAeBe systems. As discussed in Sect. 1, despite the differences in radii, magnetic field strengths and mass accretion rates between $\mathrm{HAeBe}$ 


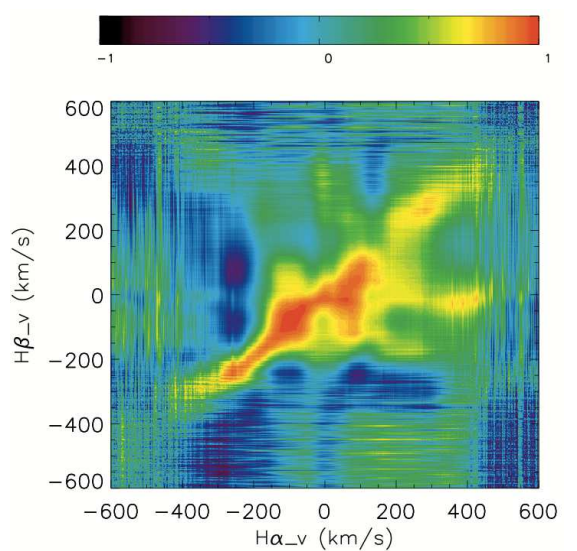

(a)

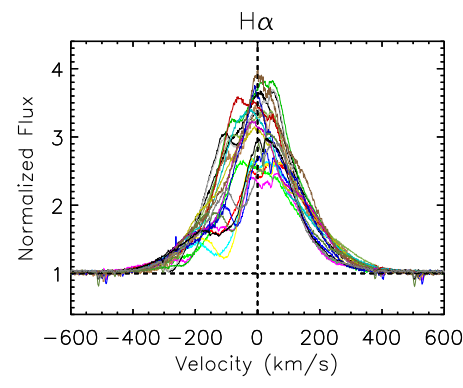

(b)

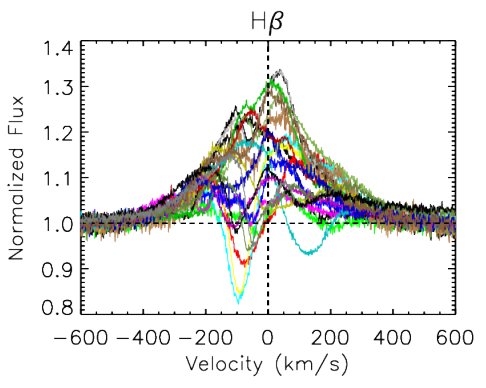

(c)

Figure 14. Correlation matrix (a) between $\mathrm{H} \alpha$ (b) and $\mathrm{H} \beta$ (c) of HD 261941. The color bar above the matrix indicates the level of correlation.

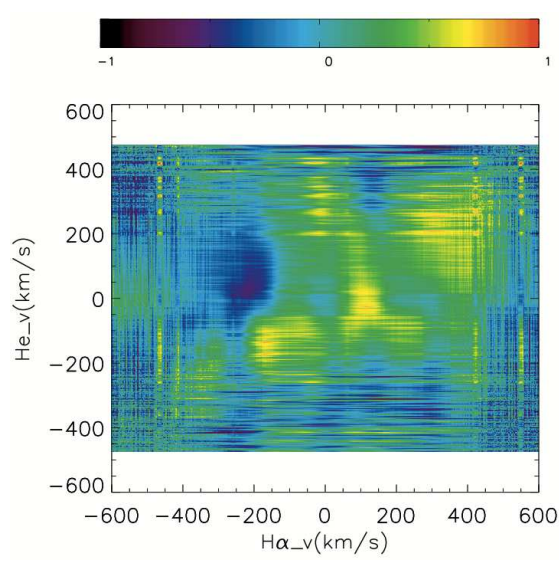

(a)

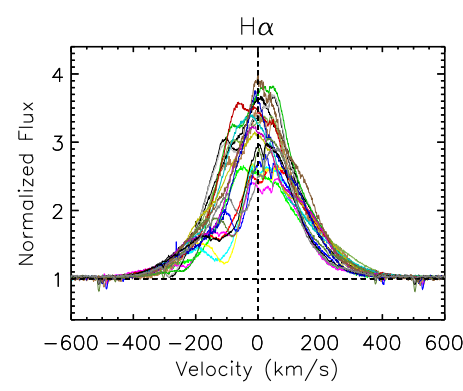

(b)

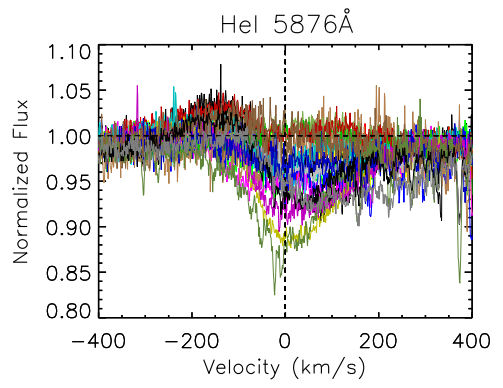

(c)

Figure 15. Correlation matrix (a) between $\mathrm{H} \alpha$ (b) and HeI $\lambda 5875.7$ (c) of HD 261941. The color bar above the matrix indicates the level of correlation.

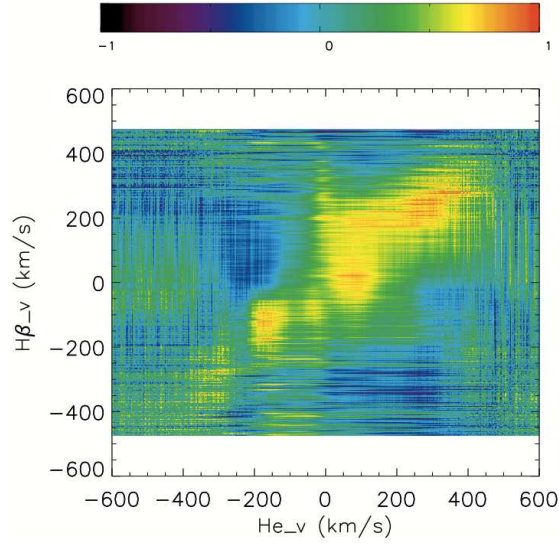

(a)

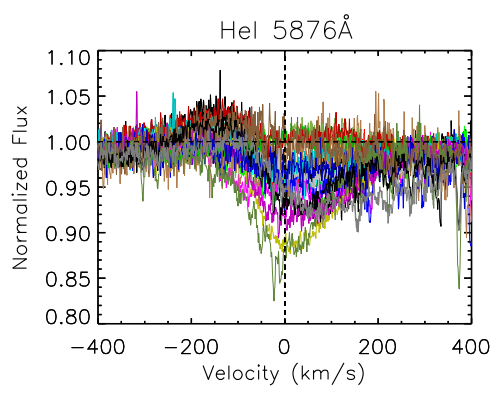

(b)

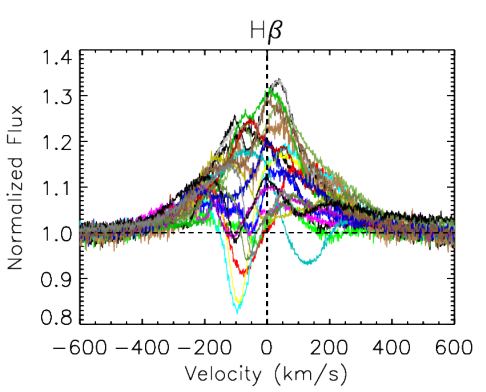

(c)

Figure 16. Correlation matrix (a) between HeI $\lambda 5875.7$ (b) and $\mathrm{H} \beta$ (c) of HD 261941. The color bar above the matrix indicates the level of correlation.

stars and T Tauri stars, magnetospheric accretion remains a possible scenario for the former. We therefore tried to apply the magnetospheric accretion model of Lima et al. $(2010, C V)$ to reproduce the observed $\mathrm{H} \alpha$ emission of V590 Mon and HD 261941.

The model is composed of a star, a magnetosphere, an accre- tion disc, and a disc wind (Fig. 23). The stellar magnetic field is described by an axisymmetric dipole. The accretion disc is considered opaque, with finite thickness and its emission component is neglected in the optical. The stellar surface is divided into a photosphere and a shock region, both emitting as blackbodies. The ac- 


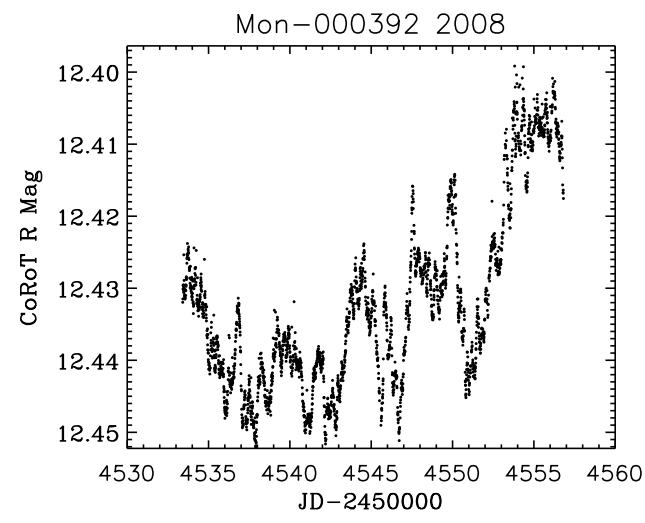

(a)

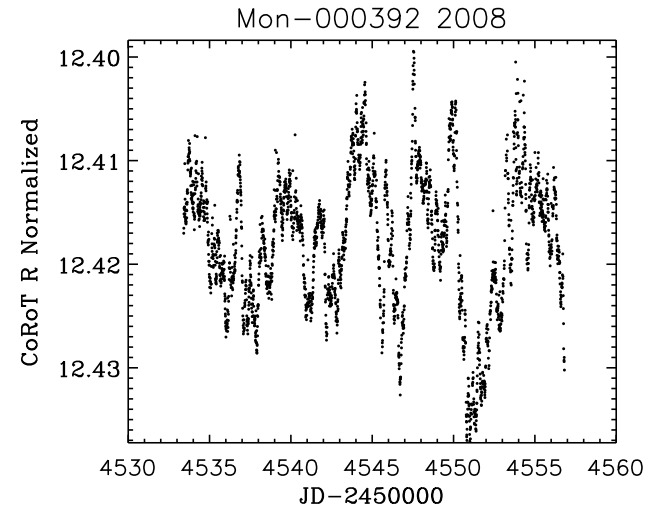

(d)

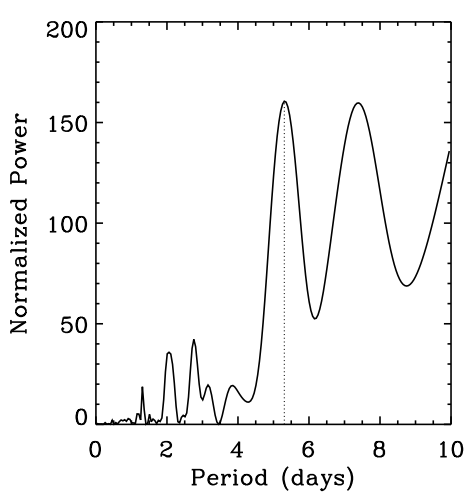

(b)

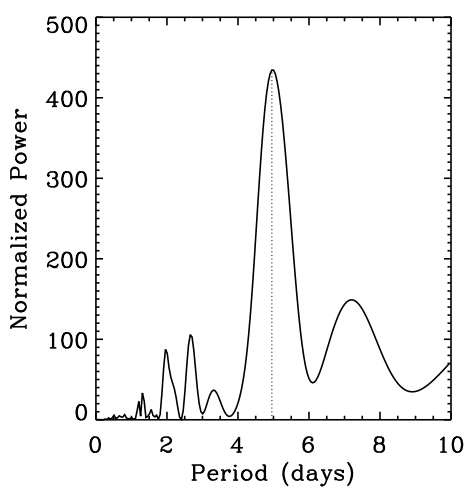

(e)

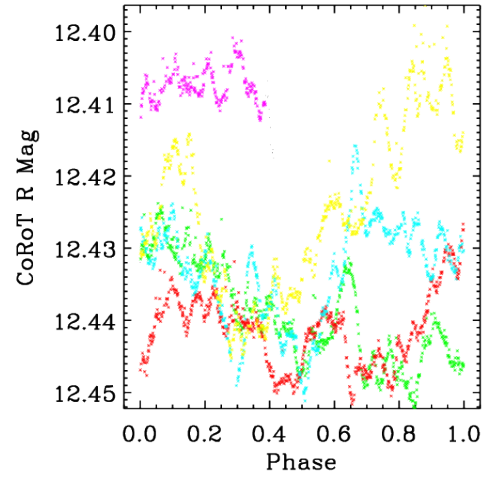

(c)

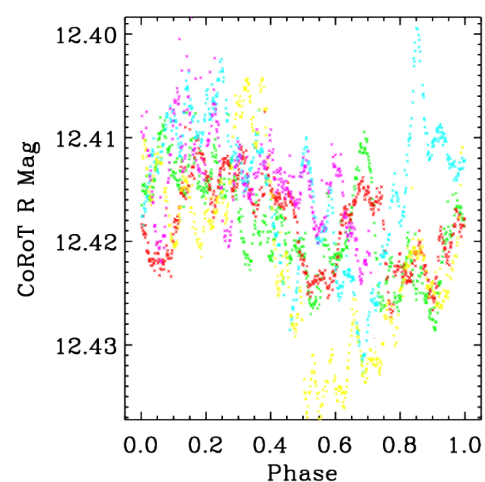

(f)

Figure 17. (a) V590 Mon CoRoT light curve with the CoRoT magnitudes calibrated using the R filter with a zero point of 26.74 mag, as described in Cody et al. (2014). The periodogram of the 2008 light curve is shown in (b) and the light curve folded in phase with the 5.3-day period in (c). The normalized CoRoT light curve is presented in (d), its corresponding periodogram is shown in (e) and the light curve folded in phase with the 5.0-day period corresponds to (f). Different colors represent different rotational cycles.
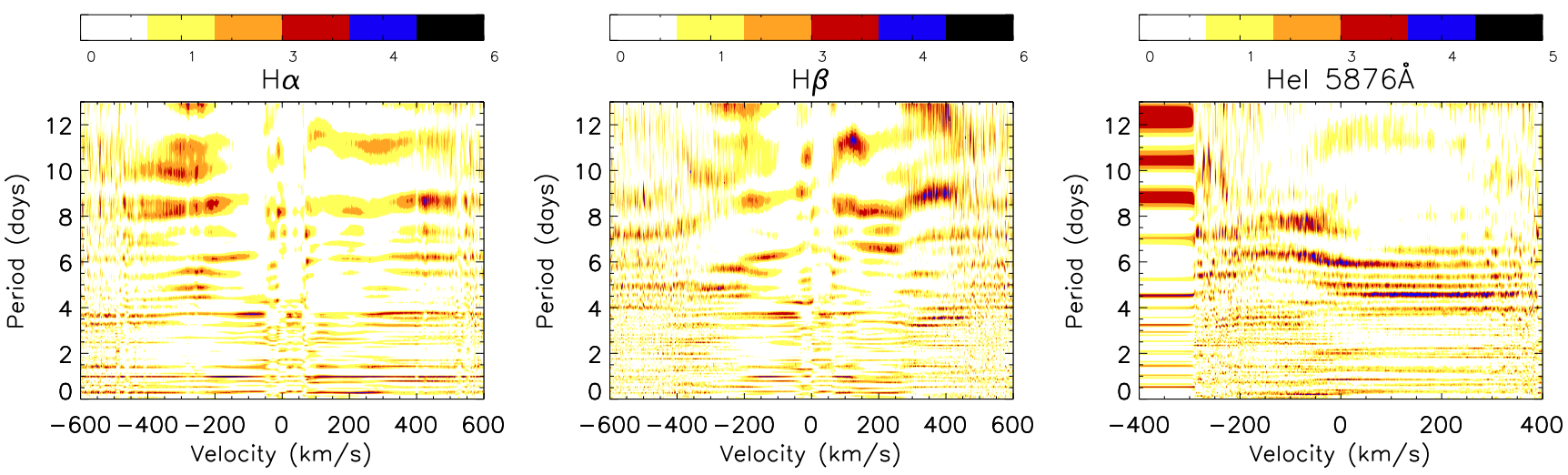

Figure 18. Periodograms of the $\mathrm{H} \alpha, \mathrm{H} \beta$ and HeI $\lambda 5875.7$ circumstellar lines of V590 Mon. The color bar represents the periodogram power. There is no significant periodicity in the $\mathrm{H} \alpha$ and $\mathrm{H} \beta$ lines. There is a period detection of about 4.2 days in the $\mathrm{HeI} \lambda 5875.7$ from 0 to $400 \mathrm{~km} \mathrm{~s}^{-1}$. 


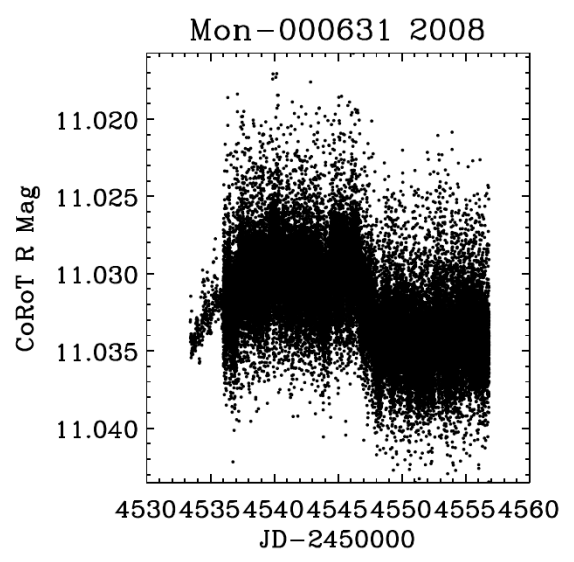

(a)

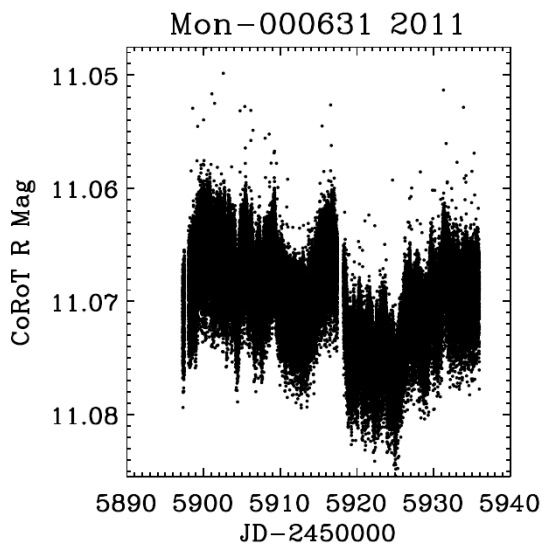

(d)

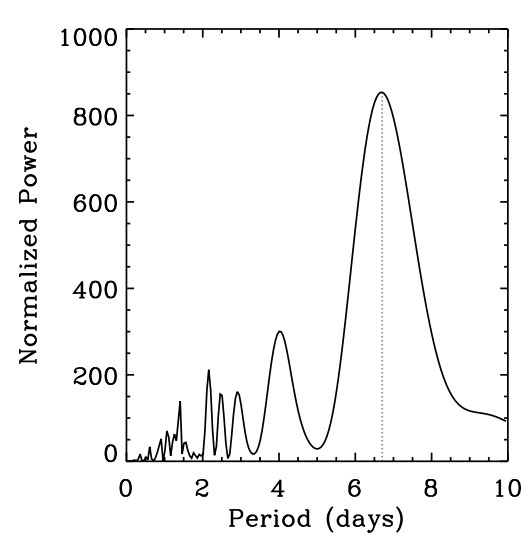

(b)

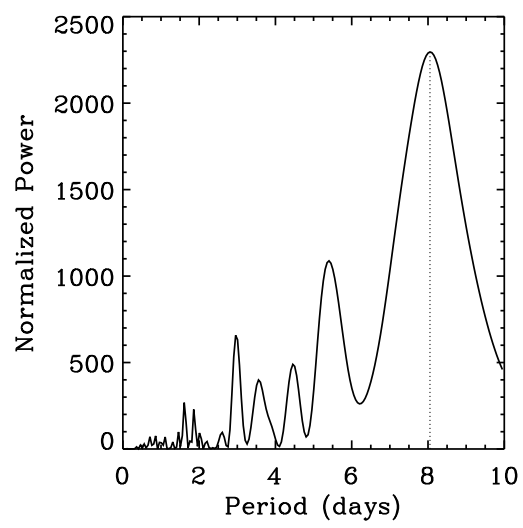

(e)

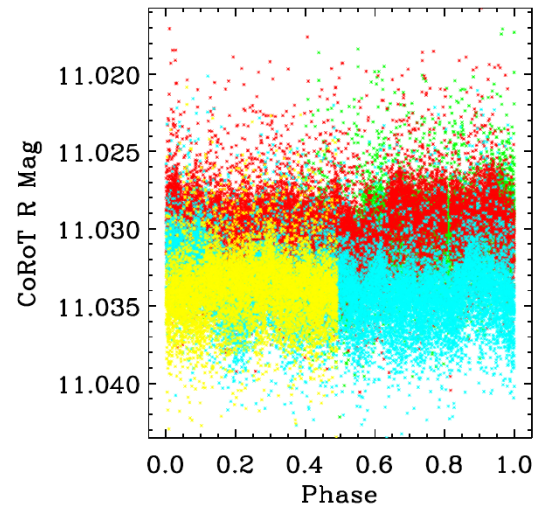

(c)

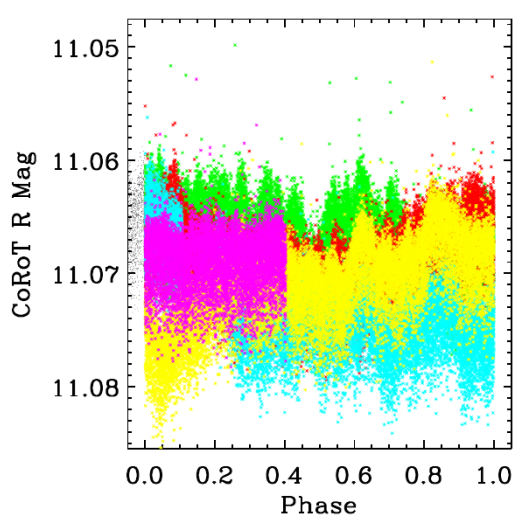

(f)

Figure 19. HD 261941 CoRoT light curves from 2008 (a) and 2011 (d). The CoRoT magnitudes were calibrated using the R filter with a zero point of 26.74 mag, as described in Cody et al. (2014). The periodograms of the 2008 and 2011 light curves are shown in (b) and (e) and the phase-folded CoRoT curves at the detected periods are presented in (c) and (f). Each color represents a different rotational cycle.
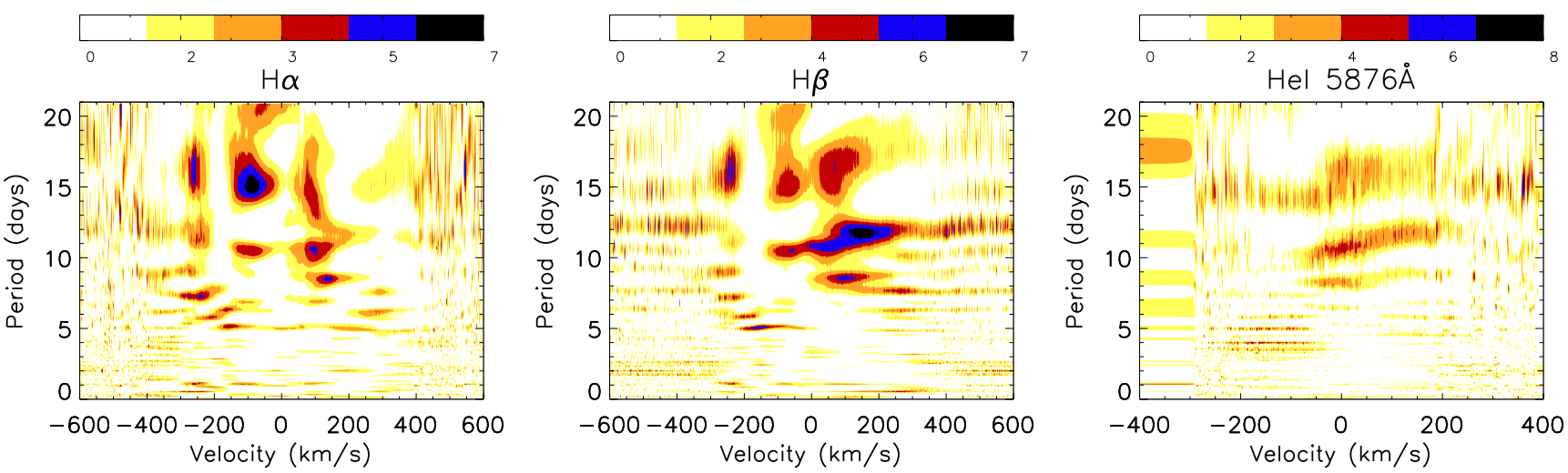

Figure 20. Periodograms of the $\mathrm{H} \alpha, \mathrm{H} \beta$ and HeI $\lambda 5875.7$ circumstellar lines of HD 261941. The color bar represents the periodogram power. There are indications of periodicities in the $\mathrm{H} \alpha$ and $\mathrm{H} \beta$ lines. 

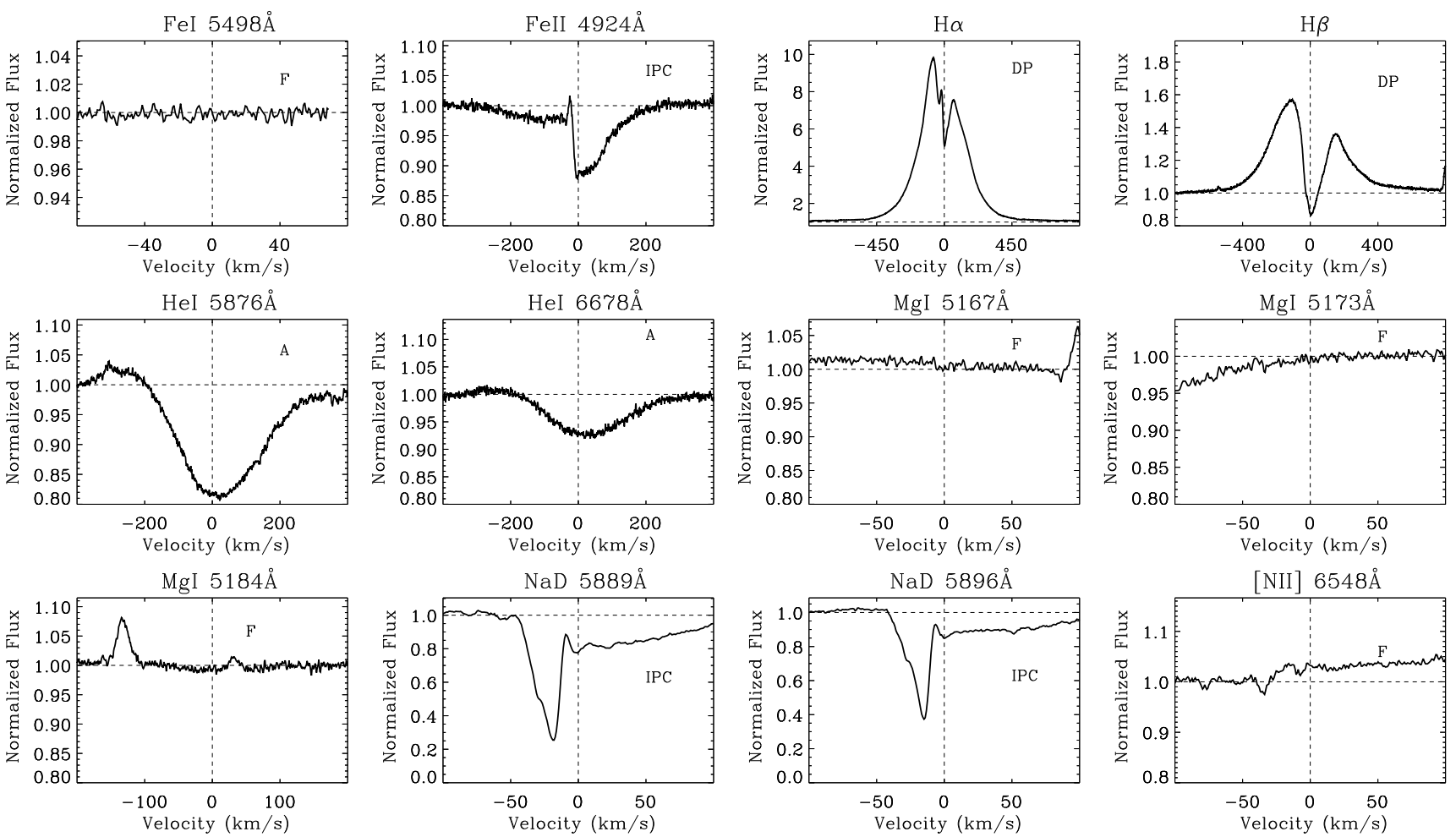

[NII] $6548 \AA$
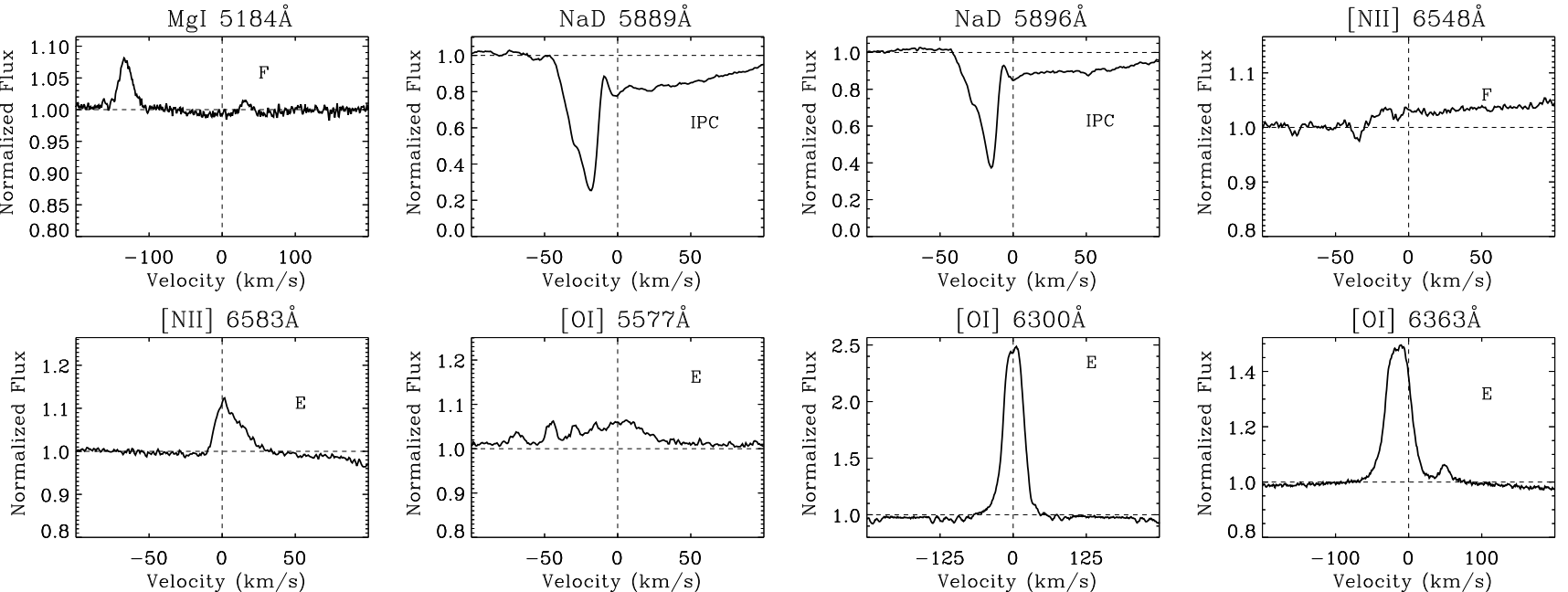

[OI] $6363 \AA$

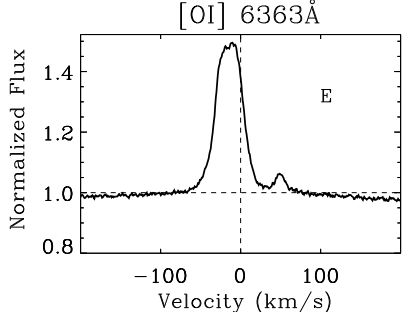

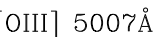

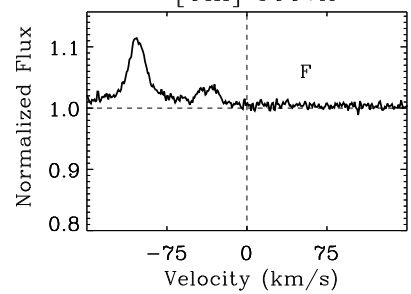

Figure 21. Average circumstelar line profiles of V590 Mon. The continuum corresponds to the horizontal dashed line and the stellar rest velocity is represented by a vertical dashed line. The profile classification is given on the right upper corner of each plot.

cretion funnel, or magnetosphere, is bounded by the magnetic field lines that intersect the disc between the inner and outer magnetospheric radii, $r_{m i}$ and $r_{m o}$, respectively. The truncation radius $\left(\mathrm{R}_{T}\right)$ corresponds to $r_{m i}$. The disc wind is limited by $r_{d i}$ and $r_{d o}$, the inner and outer wind radii, respectively, and the disc wind launching angle is given by $\vartheta_{0}$. Other important parameters of the code are the maximum magnetosphere temperature $\left(T_{\text {mag }}\right)$, the maximum disc wind temperature $\left(T_{\text {wind }}\right)$, the inclination of the system with respect to our line of sight $(i)$, the mass accretion rate $\left(\dot{M}_{a c c}\right)$ and the mass loss rate $\left(\dot{M}_{\text {loss }}\right)$. We kept the ratio between the mass loss rate and mass accretion rate at 0.1, following Pelletier \& Pudritz (1992).

Muzerolle et al. (2001) have shown that rotation has a negligible effect in the computed magnetospheric accretion line profiles when $\operatorname{vsin} i \lesssim 25 \mathrm{~km} \mathrm{~s}^{-1}$. Stars that rotate faster, however, present a toroidal velocity component in the magnetosphere that affects the shape of the line profiles and, in those cases, rotation must be included in the calculations. The $C V$ code from Lima et al. (2010) was originally developed for CTTS, which normally rotate slowly, and does not include rotation in the magnetosphere. The disc wind component, however, takes rotation into account and the outflow velocity is described by toroidal and poloidal components (Lima et al. 2010). As can be seen in Fig. 24, line profiles from disc-wind models without rotation (red lines) are strongly peaked near the stellar rest velocity. When rotation is included (blue lines), the flux at low velocities is redistributed across the emission line profile. Our targets are fast rotators, presenting $v \sin i \gtrsim 100 \mathrm{~km} \mathrm{~s}^{-1}$, and we therefore started our model calculations taking into account only 

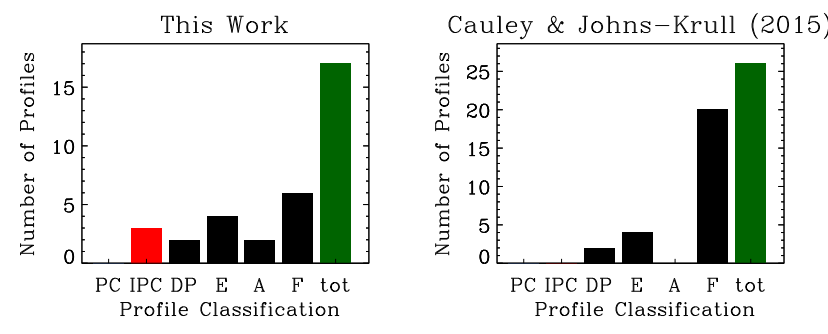

Figure 22. Histogram with the classification of the average circumstellar lines of V590 Mon with our data (left) and the classification of 26 circumstellar lines of V590 Mon by Cauley \& Johns-Krull (2015). Green represents the total number of lines analysed, red corresponds to the number of detected inverse P-Cygni profiles (IPC), and black represents doublepeaked (DP), single-peaked emission (E), absorption (A), and featureless (F) profiles detected.

Table 2. Morphological classification of the observed circumstellar lines of HD 261941 in each observed night.

\begin{tabular}{ccccccc}
\hline Line ID & PC & IPC & DP & E & A & F \\
\hline \hline $\mathrm{H} \alpha$ & 0 & 0 & 8 & 13 & 0 & 0 \\
$\mathrm{H} \beta$ & 3 & 2 & 7 & 6 & 0 & 3 \\
FeI $\lambda 5498$ & 0 & 0 & 0 & 0 & 0 & 21 \\
FeII $\lambda 4924$ & 0 & 0 & 0 & 0 & 0 & 21 \\
$\mathrm{HeI} \lambda 5876$ & 0 & 0 & 0 & 0 & 9 & 12 \\
$\mathrm{HeI} \lambda 6678$ & 0 & 0 & 0 & 0 & 0 & 21 \\
$\mathrm{MgI} \lambda 5167$ & 0 & 0 & 0 & 0 & 0 & 21 \\
$\mathrm{MgI} \lambda 5172$ & 0 & 0 & 0 & 0 & 0 & 21 \\
$\mathrm{MgI} \lambda 5184$ & 0 & 0 & 0 & 0 & 0 & 21 \\
{$[\mathrm{NII}] \lambda 6548$} & 0 & 0 & 0 & 0 & 0 & 21 \\
{$[\mathrm{NII}] \lambda 6583$} & 0 & 0 & 0 & 0 & 0 & 21 \\
{$[\mathrm{OIII}] \lambda 5007$} & 0 & 0 & 0 & 0 & 0 & 21 \\
{$[\mathrm{OI}] \lambda 6300$} & 0 & 0 & 0 & 0 & 0 & 21 \\
{$[\mathrm{OI}] \lambda 6363$} & 0 & 0 & 0 & 0 & 0 & 21 \\
$\mathrm{NaDI} \lambda 5889$ & 0 & 0 & 0 & 0 & 21 & 0 \\
$\mathrm{NaDI} \lambda 5896$ & 0 & 0 & 0 & 0 & 21 & 0 \\
\hline
\end{tabular}

the disc wind contribution that properly includes the effects of rotation.

We checked how each input parameter changed the $\mathrm{H} \alpha$ profile, affecting the line intensity, width, and morphology and we compared our results with the observed profiles. The effective temperature, the stellar mass and the radius were fixed input parameters at the values described in Tab. 1 for both systems, V590 Mon and HD 261941. Masses and radii were obtained from the CESTAM evolutionary model. The corotation radius was calculated using the formalism described in Mendigutía et al. (2011b), and this value was used as an upper boundary for the truncation radius, $\mathrm{R}_{T}$, in the $C V$ modeling. The inner disc wind radius, $r_{d i}$, started immediately after the corotation radius.

Figure A1 shows that the $\mathrm{H} \alpha$ profiles of V590 Mon do not vary much in morphology and intensity during our observations, implying that they come from an extended region that remains about the same in the timescale of our observations. The $\mathrm{H} \alpha$ correlation matrix (Fig. 11) also showed that most of the line is correlated, indicating that its variability is probably dominated by a single physical region, like the magnetosphere or a disc wind.

Several authors have analysed disc wind emission with various configurations around CTTS and HAeBe systems. Shu et al. (1994)

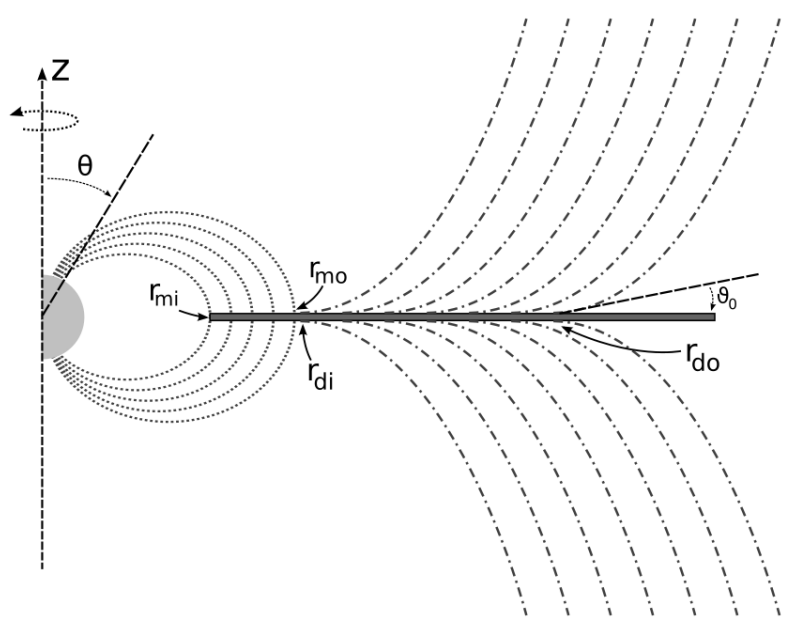

Figure 23. Sketch of the geometry used by the $C V$ model. The dotted lines represent the magnetosphere dipole field, which is limited by the inner $r_{m i}$ and outer $r_{m o}$ radii. The disc wind boundaries are defined by $r_{d i}$ and $r_{d o}$ and are shown as dash-dotted lines. The star corresponds to the gray half-circle, the disc is represented by the horizontal plane and the disc wind launching angle is $\vartheta_{0}$. Figure taken from Lima et al. (2010).

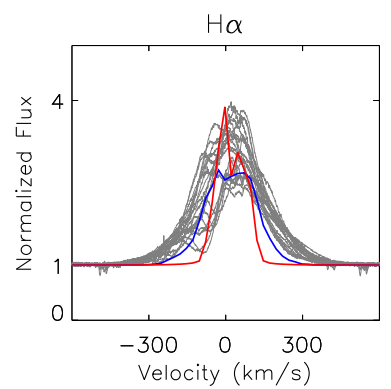

(a)

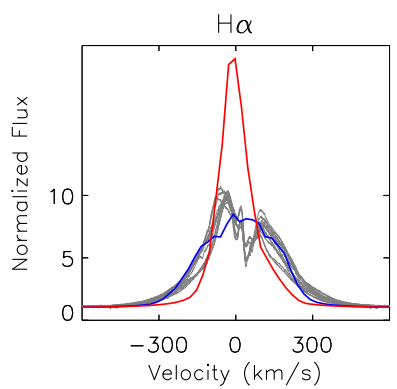

(b)
Figure 24. $\mathrm{H} \alpha$ disc-wind line profiles calculated with (blue) and without (red) rotation for HD 261941 (a) and V590 Mon (b).

Table 3. disc-wind $C V$ models calculated with the stellar parameters of V590 Mon. Models 1, 2 and 3 correspond to Figs. 25a, 25b and 25c.

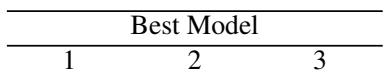

\begin{tabular}{|c|c|c|c|}
\hline Parameters & & & \\
\hline $\mathrm{M}_{*}\left[\mathrm{M}_{\odot}\right]$ & 3.2 & 3.2 & 3.2 \\
\hline $\mathrm{R}_{*}\left[\mathrm{R}_{\odot}\right]$ & 2.15 & 2.15 & 2.15 \\
\hline $\mathrm{i}\left[{ }^{\circ}\right]$ & 40 & 51 & 55 \\
\hline $\mathrm{R}_{d i}\left[\mathrm{R}_{*}\right]$ & 2.00 & 1.90 & 2.00 \\
\hline $\mathrm{R}_{d o}\left[\mathrm{R}_{*}\right]$ & 10.00 & 10.00 & 10.00 \\
\hline$\vartheta_{0}\left[{ }^{\circ}\right]$ & 53 & 53 & 53 \\
\hline$\rho\left[\mathrm{g} \mathrm{cm}^{-3}\right]$ & $6.0 \mathrm{e}-10$ & $5.9 \mathrm{e}-10$ & $6.0 \mathrm{e}-10$ \\
\hline $\mathrm{T}_{\text {wind }}[\mathrm{K}]$ & 11600 & 11600 & 11600 \\
\hline$\dot{M}_{\text {lost }}\left[\mathrm{M}_{\odot} \mathrm{yr}^{-1}\right]$ & $6.0 \mathrm{e}-8$ & $6.3 e-8$ & $6.0 \mathrm{e}-8$ \\
\hline
\end{tabular}




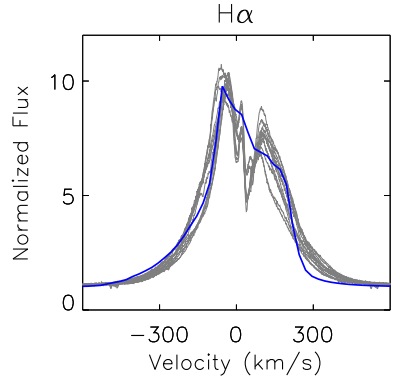

(a) Model 1

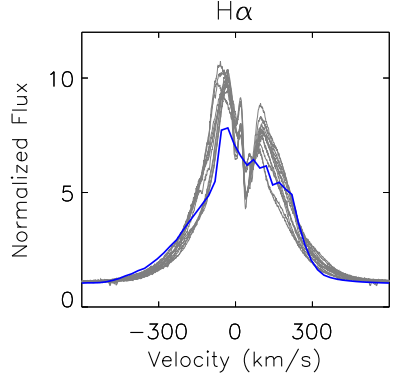

(b) Model 2

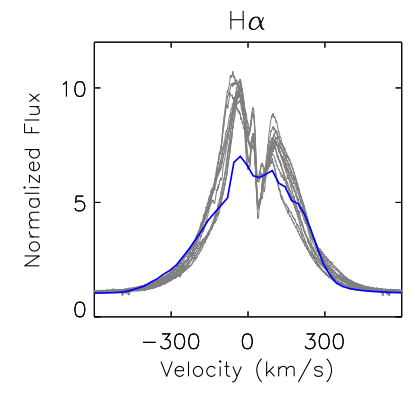

(c) Model 3

Figure 25. $\mathrm{H} \alpha$ profile of V590 Mon. The observed profiles are shown in grey. The blue lines show the theoretical line profiles from models 1,2 and 3 . They correspond to disc-wind models and the parameters of each model are listed in Tab. 3.

proposed $\mathrm{X}$-winds originating close to the corotation radius and found for CTTS wind densities around $\sim 4 \times 10^{-11} \mathrm{~g} \mathrm{~cm}^{-3}$ for massloss rates of $10^{-8} \mathrm{M}_{\odot} / \mathrm{yr}$. Drew et al. (1998) modeled the outflow of a massive $\left(\mathrm{M}_{*}=10 \mathrm{M}_{\odot}\right) \mathrm{HBe}$ system and obtained disc wind densities of $\rho_{0}=10^{-8} \mathrm{~g} \mathrm{~cm}^{-3}$, for a mass-loss rate of $10^{-7} \mathrm{M}_{\odot} / \mathrm{yr}$. More recently, Turner et al. (2014) modeled disc atmospheres of magnetically supported $\mathrm{HAeBe}$ systems with density profiles that varied from $10^{-9}$ to $10^{-14} \mathrm{~g} \mathrm{~cm}^{-3}$ with respect to the disc radius (from 0 to $0.3 \mathrm{AU}$ ).

As can be seen in Fig. 25, a dense disc wind $\left(\rho_{0} \sim 6 \times 10^{-10}\right.$ $\mathrm{g} \mathrm{cm}^{-3}$ ) is able to reproduce the emission part of the observed $\mathrm{H} \alpha$ profile of V590 Mon. The parameters used in the calculations are listed in Tab. 3. Disc winds come from larger disc radii and cover a larger emission region than magnetospheres and are expected therefore to vary on longer timescales, as observed in the $\mathrm{H} \alpha$ profiles of V590 Mon. Dense disc winds also need high outflow rates, as commonly measured in Herbig Be stars, such as V590 Mon. Our best SED model of V590 Mon, discussed in Sect. 4.1, included an envelope to reproduce the strong observed infrared excess. This indicates the presence of a large scale structure around the central object that could also be associated with a dense disc wind. Given the above arguments, we suggest that most of the $\mathrm{H} \alpha$ emission of V590 Mon comes from a dense disc wind originated in the inner accretion disc. The best model fits to the $\mathrm{H} \alpha$ line correspond to a moderate inclination of the system with respect to our line of sight $\left(i=40-55^{\circ}\right)$, which agrees with the inclination of $\mathrm{i} \sim 51^{\circ} \mathrm{ob}-$ tained in Section 6.3, while Strom et al. (1972b); Sitko et al. (1984); Rydgren \& Vrba (1987) suggested that the system is seen at high inclination due to its strong infrared SED emission.

Magnetic fields are needed to drive both disc wind outflow and magnetospheric accretion (Blandford \& Payne 1982). Assum- ing the presence of magnetised winds, it is reasonable to suppose that a stellar magnetosphere may also be present inside the disc truncation radius. The magnetic field required to sustain a magnetosphere can be estimated using the relation between the disc truncation radius and several stellar parameters as derived by Bessolaz et al. (2008):

$r_{m i}\left(R_{*}\right)=2 m_{s}^{2 / 7} B_{*}^{4 / 7} \dot{M}_{a c c}^{-2 / 7} M_{*}^{-1 / 7} R_{*}^{5 / 7}$,

where $m_{s} \sim 1$ is the sonic Mach number and $B_{*}$ is the dipolar magnetic field at the stellar equator. The disc truncation radius, $r_{m i}$, was taken as the corotation radius, and the mass accretion rate $\dot{M}_{a c c}$ of each system was obtained from the $\mathrm{H} \alpha$ line modeling. The stellar mass, $M_{*}$ and stellar radius, $R_{*}$, of V590 Mon are listed in Tab. 1 . The dipolar magnetic field necessary to sustain a magnetosphere that reaches the corotation radius in the V590 Mon system is $\mathrm{B}_{*} \sim$ $1200 \mathrm{G}$, obtained using $\mathrm{R}_{T} \sim 2.0 \mathrm{R}_{\odot}$ e $\dot{M}_{a c c} \sim 6.0 \times 10^{-7} \mathrm{M}_{\odot} \mathrm{yr}^{-1}$. However, Herbig Be stars do not generally present such strong magnetic fields (Villebrun et al. 2019). Most magnetic fields detected in HBe systems are actually below $300 \mathrm{G}$ (Wade et al. 2007; Hubrig et al. 2009, 2011; Alecian et al. 2013; Hubrig et al. 2013). We therefore decided not to take into account the magnetospheric contribution to the $\mathrm{H} \alpha$ line profile of V590 Mon.

Our second target, HD 261941, presents H $\alpha$ profiles that show significant variation in intensity and morphology in a day timescale (Fig. A2). In the most intense profiles no absorptions are observed, and the blueshifted absorptions become visible as the emission intensity decreases.

Figure 26 shows the best model fits obtained for HD 261941 with the disc wind component, calculated with the parameters listed in Tab. 4. Using a disc-wind model, we could not reproduce the main characteristics of the observed H $\alpha$ profiles of HD 261941. Models 1 and 2 (Fig. 26a and b) show intensities which are similar only to the less intense observed profiles and the theoretical profiles do not present blueshifted absorptions. Part of the observed $\mathrm{H} \alpha$ profiles may then originate in a region different from a disc wind, such as a magnetosphere. Due to that, we decided to compute hybrid models that consistently calculate magnetosphere and disc wind components. Unfortunately rotation is not implemented in the magnetosphere calculations of our $C V$ code version and we must keep that in mind when interpreting the theoretical line profile, since the inclusion of rotation in the magnetospheric calculations would tend to produce less centrally peaked profiles, as shown Muzerolle et al. (2001). With the hybrid model we were able to obtain a better match to the observed $\mathrm{H} \alpha$ profiles, as shown in Fig. 27. The model calculations were performed with the parameters listed in Tab. 5 and the results point to a significant contribution from a magnetosphere to the line flux in HD 261941. The intensity of the stellar magnetic field that corresponds to the magnetosphere used in the hybrid model, according to Eq. 9 and the parameters listed in Tab. 5, is of $\sim 519 \mathrm{G}$. Magnetic fields around $1 \mathrm{kG}$ were detected through spectropolarimetry in HAe systems (Hubrig et al. 2013), giving support to our model results. The system inclination of $i \sim$ $79^{\circ}$ calculated in Section 6.3 for HD 261941 agrees with the inclinations obtained with both the hybrid $\left(i=77^{\circ}\right)$ and disc-wind $\left(i=75-85^{\circ}\right)$ models.

The impact of rotation on the magnetospheric contribution is significant and needs to be thought about. Due to rotation, the flux in the emission line is redistributed from line center to a range of velocities and, as vsin $i$ increases, the corotation radius decreases, leading to small accretion flows, since accretion can only occur inside corotation. Muzerolle et al. (2004) have computed magneto- 


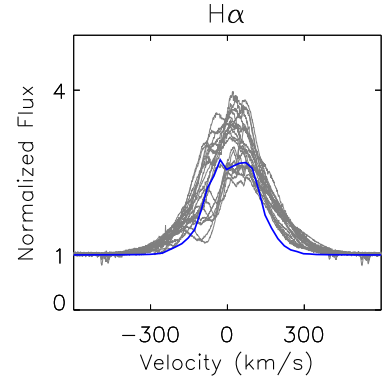

(a) Model 1

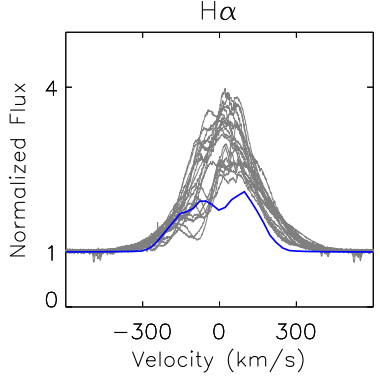

(b) Model 2
Figure 26. $\mathrm{H} \alpha$ profiles of HD 261941. The observed profiles are shown in grey. The two disc-wind models (blue lines) were calculated with the parameters listed in Tab. 4.

Table 4. Parameters of the best CV model fits of the H $\alpha$ line of HD 261941 with a disc-wind model. Models 1 and 2 correspond to Figs. 26a and 26b

$$
\begin{array}{rr}
\hline \multicolumn{2}{l}{\text { Best Models }} \\
\hline 1
\end{array}
$$

\begin{tabular}{ccc}
\hline \hline \multicolumn{2}{c}{ Parameters } & \\
\hline $\mathrm{M}_{*}\left[\mathrm{M}_{\odot}\right]$ & 2.3 & 2.3 \\
\hline $\mathrm{R}_{*}\left[\mathrm{R}_{\odot}\right]$ & 2.9 & 2.9 \\
\hline $\mathrm{i}\left[{ }^{\circ}\right]$ & 85 & 75 \\
\hline $\mathrm{R}_{d i}\left[\mathrm{R}_{*}\right]$ & 1.8 & 2.8 \\
\hline $\mathrm{R}_{d o}\left[\mathrm{R}_{*}\right]$ & 10.00 & 10.00 \\
\hline$\vartheta_{0}\left[{ }^{\circ}\right]$ & 14.57 & 33.42 \\
\hline$\rho\left[\mathrm{g} \mathrm{cm}^{-3}\right]$ & $7.8 \mathrm{e}-11$ & $1.2 \mathrm{e}-10$ \\
\hline $\mathrm{T}_{\text {wind }}[\mathrm{K}]$ & 9200 & 9600 \\
\hline$\dot{M}_{\text {lost }}\left[\mathrm{M}_{\odot} \mathrm{yr}^{-1}\right]$ & $3.0 \mathrm{e}-8$ & $3.0 \mathrm{e}-8$ \\
\hline
\end{tabular}

spheric accretion models of rapidly rotating young stars (up to 140 $\mathrm{km} \mathrm{s}^{-1}$ ) and showed that small corotation radii imply Balmer line profiles that differ from the commonly observed ones. They suggested that the magnetic field geometry of HAeBe systems may be different from CTTS and therefore the simple dipolar geometry, like the one used in our models and in most magnetospheric accretion models in the literature, may not be adequate to compute line profiles for intermediate mass systems. The implementation of a more complex magnetic field geometry in the $C V$ code is however beyond the scope of the present analysis.

Giving support to our preferred hybrid model of the H $\alpha$ profile of HD 261941, there is evidence in the literature that Herbig Ae stars are undergoing accretion. Garcia Lopez et al. (2006) computed mass accretion rates for a sample of $36 \mathrm{HAe}$ stars from the $\operatorname{Br} \gamma$ line luminosity and showed that $80 \%$ of their sample presented evidence of accretion from a circumstellar disc with mass accretion rates in the range $3 \times 10^{-9} \lesssim \dot{M}_{a c c} \lesssim 10^{-6} \mathrm{M}_{\odot} \mathrm{yr}^{-1}$. Muzerolle et al. (2004) analysed the HAe system UX Ori (A2 spectral type; $\mathrm{M}_{*}=3 \mathrm{M}_{\odot} ; \mathrm{R}_{*}=3 \mathrm{R}_{\odot} ; 70<v \sin i<140 \mathrm{~km} \mathrm{~s}^{-1} ; \dot{M}_{a c c} \sim$ $\left.10^{-8} \mathrm{M}_{\odot} \mathrm{yr}^{-1} ; i=75^{\circ}\right)$ and showed that the magnetospheric accretion model could reproduce the observed emission line profiles, implying that at least some HAe stars might present magnetospheric

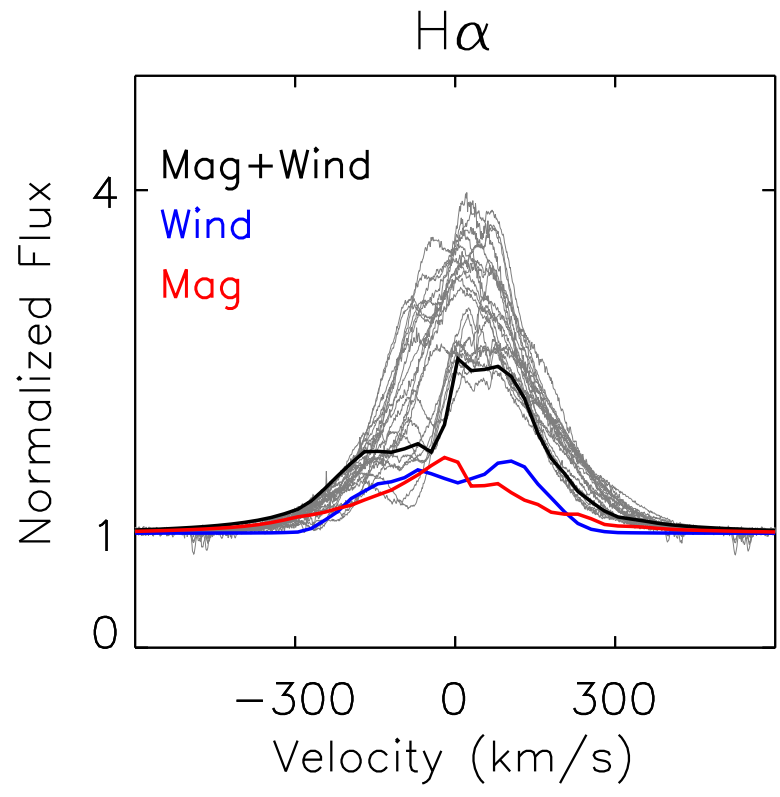

Figure 27. H $\alpha$ profiles of HD 261941 computed with a hybrid model. The observed profiles are shown in grey. The disc-wind model is represented by a blue line, the magnetospheric contribution is shown as a red line and the resulting hybrid profile is shown in black. The parameters used in the calculations are listed in Tab. 5.

Table 5. Parameters of the best CV model fits of the H $\alpha$ line of HD 261941 with a hybrid (magnetosphere and disc-wind) model

\begin{tabular}{cc}
\hline Parameters & Values \\
\hline \hline $\mathrm{M}_{*}\left[\mathrm{M}_{\odot}\right]$ & 2.3 \\
\hline $\mathrm{R}_{*}\left[\mathrm{R}_{\odot}\right]$ & 2.9 \\
\hline $\mathrm{i}\left[{ }^{\circ}\right]$ & 77 \\
\hline $\mathrm{R}_{m i}\left[\mathrm{R}_{*}\right]$ & 2.0 \\
\hline $\mathrm{R}_{m o}\left[\mathrm{R}_{*}\right]$ & 2.45 \\
\hline $\mathrm{R}_{d i}\left[\mathrm{R}_{*}\right]$ & 2.50 \\
\hline $\mathrm{R}_{d o}\left[\mathrm{R}_{*}\right]$ & 10.00 \\
\hline$\vartheta_{0}\left[{ }^{\circ}\right]$ & 31.80 \\
\hline$\rho\left[\mathrm{g} \mathrm{cm}{ }^{-3}\right]$ & $1.1 \mathrm{e}-10$ \\
\hline $\mathrm{T}_{m a g}[\mathrm{~K}]$ & 8200 \\
\hline $\mathrm{T}_{\text {wind }}[\mathrm{K}]$ & 8900 \\
\hline$\dot{M}_{\text {acc }}\left[\mathrm{M}_{\odot} \mathrm{yr} \mathrm{r}^{-1}\right]$ & $2.8 \mathrm{e}-7$ \\
\hline$\dot{M}_{\text {lost }}\left[\mathrm{M}_{\odot} \mathrm{yr}{ }^{-1}\right]$ & $2.8 \mathrm{e}-8$ \\
\hline
\end{tabular}

accretion. Rousselet-Perraut et al. (2010) reproduced the $\mathrm{H} \alpha$ profile of the HAe system AB Aur with a hybrid magnetosphere and wind model. Mendigutía et al. (2017) analysed two HAe systems and were able to model the $\mathrm{H} \alpha$ line of HD 179218 with a magnetospheric accretion code, but they could not reproduce the $\mathrm{H} \alpha$ 
profiles of HD 141569 due to its high rotational velocity $(v \sin i \sim$ $258 \mathrm{~km} \mathrm{~s}^{-1}$ ).

Accretion and outflow are complex processes, which depend on the star-disc interaction, the structure of the stellar and disc magnetic fields, accretion disc properties, and mass accretion rate, among many other parameters (Romanova et al. 2003, 2004; Romanova \& Owocki 2015). Considering the limitations of the $C V$ model and the complexity of the accretion and ejection processes in young systems, we were able to reasonably reproduce the $\mathrm{H} \alpha$ line profile of V590 Mon with a rotating disc-wind MHD model. The disc-wind model however did not reproduce the observed $\mathrm{H} \alpha$ profile of HD 261941. Hybrid models that consistently take into account the contribution of a magnetosphere and a disc wind resulted in line profiles that were a better match to the observations with the caveat that the magnetosphere in our model is non-rotating.

\section{DISCUSSION AND CONCLUSION}

We analysed spectroscopic and photometric synoptic data of two $\mathrm{HAeBe}$ stars with similar ages and different internal structures, as shown in the HR diagram of Fig. 1. V590 Mon is a Herbig Be system and HD 261941, a Herbig Ae system that belong to the young stellar cluster NGC 2264. Both systems present substantial IR flux excess, as can be seen in their SEDs (Fig. 2), showing that they are still surrounded by a significant amount of circumstellar material from which they can accrete. We studied the star-disc interaction through the dynamics of the accretion and outflow processes in the two systems.

Magnetospheric accretion models are widely accepted to describe the star-disc interaction and the accretion process in CTTS, but it is still unclear how accretion proceeds in HAeBe systems. These models are based on the assumption that the stellar magnetic field is strong enough to truncate the inner disc and control the accretion process from the disc to the star. Magnetic fields would also be responsible for magnetically controlled outflows in the form of stellar and disc winds.

The generation of magnetic fields in HAeBe systems, which are strong enough to truncate the inner accretion disc, is not yet understood. In low-mass stars, magnetic fields can be continuously generated due to the large plasma motions in the convective envelope (Alecian 2014). However, HAeBe systems do not present convective envelopes and only a few systems have had their magnetic fields measured so far.

Alecian (2014) proposed two possible scenarios for the origin of magnetic fields in HAeBe stars. The first one assumes that magnetic fields from the molecular clouds that originated the stars were sustained during star formation, becoming large-scale magnetic fields, slowly decaying with time. The second scenario proposes a magnetic field generated during the early convective phases that HAeBe systems go through. The fossil field theory is compatible with the large-scale topology and the magnetic field strength observed in the few magnetic HAeBe systems (Braithwaite \& Spruit 2017). An alternative for the magnetic field origin in radiative stars was proposed by Ferrario et al. (2009), in which the fossil field of a single star may be a result of merging processes. The merging could generate a differential rotation and thus amplify the original magnetic field. Schneider et al. (2019), through 3D MHD simulations, also showed that the merger of two massive $\left(>1.5 \mathrm{M}_{\odot}\right)$ stars can generate strong magnetic fields and they concluded that stellar mergers are a feasible mechanism for the origin of magnetic fields in Ap stars. Recently, Guerrero et al. (2019) investigated, through
MHD simulations, the evolution of the internal magnetic fields in radiative stars. They analysed the possibility that oscillations due to Taylor instabilities together with differential rotation could sustain a dynamo and explain the occurence of Ap/Bp systems. The occurence of magnetic fields in $\mathrm{HAeBe}$ stars remains therefore a possibility, at least theoretically.

Added to the uncertanties to generate and sustain a magnetic field in HAeBe stars, the magnetic field measurements in HAeBe systems also remain difficult due to larger rotational velocities and fewer photospheric lines in their spectra compared to the low-mass T Tauri stars. Villebrun et al. (2019), for example, analysed spectropolarimetric data from a sample of 38 intermediate-mass stars and could only obtain reliable magnetic field detections for stars with $v \sin i$ less than $80 \mathrm{~km} \mathrm{~s}^{-1}$, using the ESPaDOnS spectropolarimeter $(\mathrm{R} \sim 67,000)$. This result shows the difficulty to detect magnetic fields in fast rotating stars, like the HAeBe systems we analysed, which present $v \sin i>100 \mathrm{~km} \mathrm{~s}^{-1}$.

The spectral type boundary between magnetic and nonmagnetic systems in the pre-main sequence remains unclear. Ababakr et al. (2017) used spectropolarimetry to measure the linear polarization across the $\mathrm{H} \alpha$ line and found that around B7-B8 spectral types there was a switch between linear line polarization (like in CTTS) to depolarization, which they related to a change from magnetospheric accretion in Herbig Ae systems to boundary layer accretion in Herbig Be systems. This could explain why HD 261941 shows magnetospheric accretion features while V590 Mon does not. On the other hand, Vioque et al. (2018) found, after analysing low and high-mass young stars, that different accretion regimes tended to switch around $7 \mathrm{M}_{\odot}$. More recently, Wichittanakom et al. (2020) analysed various spectroscopic accretion properties of a sample of $163 \mathrm{Herbig} \mathrm{Ae} / \mathrm{Be}$ stars and suggested that the accretion mode changes around $4 \mathrm{M}_{\odot}$ from magnetospheric accretion in HAe systems to boundary layer in the more massive stars.

Assuming that at least some $\mathrm{HAeBe}$ systems are able to sustain a magnetic field and drive magnetospheric accretion, we would expect to see signatures of magnetospheric accretion in the spectra of these systems. Redshifted absorption components that go below the continuum are one of the telltales of magnetospheric accretion and are produced by high-velocity gas accelerated at free fall velocities in accretion flows. They are often present in the spectra of CTTS and are particularly common in high inclination systems with respect to our line of sight, since in this geometry our viewing angle crosses a large fraction of the accretion column. In several of our spectra, the $\mathrm{H} \beta$ line of HD 261941 shows redshifted absorptions below the continuum, as expected in magnetospheric accretion models, and this HAe system is seen at high inclination, according to our calculations. We do not see redshifted absorptions below the continuum in $\mathrm{H} \alpha$ in either of our targets, but this does not rule out the magnetospheric accretion scenario. According to Kurosawa \& Romanova (2013), who computed hydrogen line profiles (e.g. $\mathrm{H} \alpha, \mathrm{H} \beta, \mathrm{H} \gamma, \mathrm{H} \delta, \mathrm{Pa} \beta$ e $\mathrm{Br} \gamma$ ) as a function of rational phase for CTTS, the redshifted absorptions are more common in hydrogen lines such as $\mathrm{H} \gamma, \mathrm{H} \delta, \mathrm{Pa} \beta$ and $\mathrm{Br} \gamma$ than in $\mathrm{H} \alpha$ and $\mathrm{H} \beta$.

Classical $\mathrm{T}$ Tauri stars undergoing magnetospheric accretion normally present highly variable emission line profiles due to the rotation of an asymmetric accretion flow region. Comparing CTTS and HAe systems, Costigan et al. (2014) showed that the typical variability timescales of $\mathrm{H} \alpha$ in CTTS and HAe stars are similar, of the order of days, suggesting that accretion proceeds in the same way in this large range of masses. Mendigutía et al. (2011a) analysed the variability of circumstellar lines of a sample of $38 \mathrm{HAeBe}$ 
stars and showed that the $\mathrm{H} \alpha$ line of HBe systems tend to present stable profiles with blueshifted absorptions, indicating a possible wind origin for the line, while less massive HAe systems showed $\mathrm{H} \alpha$ profiles with significant variability and often with redshifted absorption components, pointing to a magnetospheric accretion origin for the line. Our data overall agree with these results, since HD 261941, a HAe system, presents considerable $\mathrm{H} \alpha$ profile variability in a timescale of days (Fig. A2) and redshifted absorptions below the continuum in $\mathrm{H} \beta$, while V590 Mon, a HBe system, shows $\mathrm{H} \alpha$ profiles which are much more stable in the same timescale (Fig. A1).

Taking into account all the arguments above, there is no strong evidence that V590 Mon is undergoing magnetospheric accretion, since the Balmer line profiles are rather stable on a week timescale and there is not a systematic presence of redshifted absorptions in the observed spectra. The $\mathrm{H} \alpha$ correlation matrix indicates that a single process dominates the line emission region and according to our $\mathrm{H} \alpha$ models, we suggest a dense disc wind origin for the $\mathrm{H} \alpha$ line. Our best fit models indicate that the system is seen at moderate inclinations $\left(40-55^{\circ}\right)$ and presents a large mass accretion rate $(\sim$ $\left.6.0 \times 10^{-7} \mathrm{M}_{\odot} \mathrm{yr}^{-1}\right)$.

HD 261941, on the other hand, shows evidences of magnetospheric accretion, such as strong emission line variability and redshifted absorption features at high velocities. The correlation matrices indicate the Balmer lines are formed in various regions and we could not reproduce the main characteristics of the observed $\mathrm{H} \alpha$ line of HD 261941 using a disc-wind model. We suggest that the $\mathrm{H} \alpha$ line in this system comes from both a magnetosphere and a disc wind, based on our preliminary calculations of a hybrid MHD model with a non-rotating magnetosphere. HD 261941 is therefore a good candidate to present a stellar magnetic field which is strong enough to sustain magnetospheric accretion.

Our results reinforce the idea that Herbig Ae systems may accrete through a magnetosphere, while a different mechanism governs accretion at higher masses. A detailed analysis of the variability of accretion features of a large number of intermediate-mass Pre-Main sequence stars of various spectral types is needed to draw firm conclusions on the accretion and outflow mechanisms at work in this class of systems.

\section{ACKNOWLEDGEMENTS}

TM, SHPA and APS acknowledge financial support from CNPq, CAPES and Fapemig. We thank an anonymous referee for fruitful comments that helped to improve the paper. We are grateful to Gustavo Guerrero, Pauline McGinnis and Natalia Landim for fruitful discussions and contributions. TM thanks IPAG (Institut de Planétologie et d'Astrophysique de Grenoble) for their hospitality during part of this work. This work made use of the BimMag4 code by O. Kochukov and the $S M E$ code by Jeff Valenti. This work has made use of the SIMBAD database.

\section{REFERENCES}

Ababakr K. M., Fairlamb J. R., Oudmaijer R. D., van den Ancker M. E., 2015, MNRAS, 452, 2566

Ababakr K. M., Oudmaijer R. D., Vink J. S., 2017, MNRAS, 472, 854

Alecian E., 2014, in Putting A Stars into Context: Evolution E., Stars

R., eds, Mathys, Gautier and Griffin, Elizabeth R. and Kochukhov, Oleg and Monier, Richard and Wahlgren, Glenn M. pp 84-92 (arXiv: 1310.1725)
Alecian E., et al., 2013, MNRAS, 429, 1001

Alecian E., Villebrun F., Grunhut J., Hussain G., Neiner C., Wade G. A., 2017, preprint, (arXiv: 1705.10650)

Alencar S. H. P., Batalha C., 2002, ApJ, 571, 378

Alencar S. H. P., Basri G., Hartmann L., Calvet N., 2005, A\&A, 440, 595

Bessolaz N., Zanni C., Ferreira J., Keppens R., Bouvier J., 2008, A\&A, 478, 155

Bhatt H. C., Sagar R., 1992, A\&A, 92, 473

Blandford R. D., Payne D. G., 1982, MNRAS, 199, 883

Boehler Y., Weaver E., Isella A., Ricci L., Grady C., Carpenter J., Perez L., 2017, ApJ, 840, 60

Boehler Y., et al., 2018, ApJ, 853, 162

Boehm T., Catala C., 1995, A\&A, 301, 155

Bouvier J., et al., 1999, A\&A, 349, 619

Bouvier J., Alencar S. H. P., Harries T. J., Johns-Krull C. M., Romanova M. M., 2007, Protostars and Planets V, pp 479-494

Braithwaite J., Spruit H. C., 2017, RSOS, 4, 160271

Breger M., 1972, ApJ, 171, 539

Breger M., 1974, ApJ, 188, 53

Cabrit S., Edwards S., Strom S. E., Strom K. M., 1990, ApJ, 354, 687

Castelli F., Kurucz R. L., 2004, New Grids of ATLAS9 Model Atmospheres (arXiv:astro-ph/0405087)

Cauley P. W., Johns-Krull C. M., 2014, ApJ, 797, 112

Cauley P. W., Johns-Krull C. M., 2015, ApJ, 810, 5

Clarke A. J., Oudmaijer R. D., Lumsden S. L., 2005, MNRAS, 363, 1111

Cody A. M., Stauffer J. R., Micela G., Baglin A., CSI 2264 Team 2013, Astronomische Nachrichten, 334, 63

Cody A. M., et al., 2014, AJ, 147, 82

Costigan G., Vink J. S., Scholz A., Ray T., Testi L., 2014, MNRAS, 440, 3444

Drew J. E., Proga D., Stone J. M., 1998, MNRAS, 296, L6

Dunkin S. K., Barlow M. J., Ryan S. G., 1997, MNRAS, 286, 604

Esau C. F., Harries T. J., Bouvier J., 2014, MNRAS, 443, 1022

Fairlamb J. R., Oudmaijer R. D., Mendigutía I., Ilee J. D., van den Ancker M. E., 2015, MNRAS, 453, 976

Fairlamb J. R., Oudmaijer R. D., Mendigutia I., Ilee J. D., van den Ancker M. E., 2017, MNRAS, 464, 4721

Ferrario L., Pringle J. E., Tout C. A., Wickramasinghe D. T., 2009, MNRAS, 400, L71

Finkenzeller U., Mundt R., 1984, A\&AS, 55, 109

Flaccomio E., Micela G., Sciortino S., Favata F., Corbally C., Tomaney A., 1999, A\&A, 345, 521

Flaccomio E., Micela G., Sciortino S., 2006, A\&A, 455, 903

Gaia Collaboration et al., 2016, A\&A, 595, A1

Gaia Collaboration et al., 2018, A\&A, 616, A1

Garcia Lopez R., Natta A., Testi L., Habart E., 2006, A\&A, 459, 837

Gregory S. G., Donati J.-F., Morin J., Hussain G. A. J., Mayne N. J., Hillenbrand L. A., Jardine M., 2012, ApJ, 755, 97

Guerrero G., Del Sordo F., Bonanno A., Smolarkiewicz P. K., 2019, arXiv e-prints, p. arXiv:1909.02897

Gullbring E., Hartmann L., Briceño C., Calvet N., 1998, ApJ, 492, 323

Herbig G. H., 1960, ApJS, 4, 337

Hillenbrand L. A., Strom S. E., Vrba F. J., Keene J., 1992, ApJ, 397, 613

Horne J. H., Baliunas S. L., 1986, ApJ, 302, 757

Hubrig S., et al., 2009, A\&A, 502, 283

Hubrig S., Ilyin I., Briquet M., Schöller M., González J. F., Nuñez N., De Cat P., Morel T., 2011, A\&A, 531, L20

Hubrig S., Ilyin I., Schöller M., Lo Curto G., 2013, AN, 334, 1093

Johns C. M., Basri G., 1995a, AJ, 109, 2800

Johns C. M., Basri G., 1995b, ApJ, 449, 341

Johns-Krull C. M., Valenti J. A., Koresko C., 1999, ApJ, 516, 900

Kearns K. E., Herbst W., 1998, AJ, 116, 261

Klaassen P. D., et al., 2013, A\&A, 555, A73

Kochukhov 2007, Synth3. Phtsics of Magnetic Stars

Kurosawa R., Romanova M. M., 2013, MNRAS, 431, 2673

Kurosawa R., Harries T. J., Symington N. H., 2006, MNRAS, 370, 580

Kurucz R., 1993, ATLAS9 Stellar Atmosphere Programs and 2 km/s 
grid. Kurucz CD-ROM No. 13. Cambridge, Mass.: Smithsonian Astrophysical Observatory, 1993., 13

Lamm M. H., Bailer-Jones C. A. L., Mundt R., Herbst W., Scholz A., 2004, A\&A, 417, 557

Lima G. H. R. A., Alencar S. H. P., Calvet N., Hartmann L., Muzerolle J., 2010, A\&A, 522, A104

Liu T., Zhang H., Wu Y., Qin S.-L., Miller M., 2011, APJ, 734, 22

Luri X., et al., 2018, A\&A, 616, A9

Makidon R. B., Rebull L. M., Strom S. E., Adams M. T., Patten B. M., 2004, AJ, 127, 2228

Marques J. P., et al., 2013, A\&A, 549, A74

McGinnis P., Dougados C., Alencar S. H. P., Bouvier J., Cabrit S., 2018, A\&A, 620, A87

McNamara B. J., 1976, AJ, 81, 845

Mendigutía I., Eiroa C., Montesinos B., Mora A., Oudmaijer R. D., Merín B., Meeus G., 2011a, A\&A, 529, A34

Mendigutía I., Calvet N., Montesinos B., Mora A., Muzerolle J., Eiroa C., Oudmaijer R. D., Merín B., 2011b, A\&A, 535, A99

Mendigutía I., Mora A., Montesinos B., Eiroa C., Meeus G., Merín B., Oudmaijer R. D., 2012, A\&A, 543, A59

Mendigutía I., Oudmaijer R. D., Mourard D., Muzerolle J., 2017, MNRAS, 464, 1984

Mendoza V. E. E., Gomez T., 1980, MNRAS, 190, 623

Morel P., Lebreton Y., 2008, Ap\&SS, 316, 61

Munari U., Sordo R., Castelli F., Zwitter T., 2005, A\&A, 442, 1127

Muzerolle J., Hartmann L., Calvet N., 1998, AJ, 116, 455

Muzerolle J., Calvet N., Hartmann L., 2001, ApJ, 550, 944

Muzerolle J., D’Alessio P., Calvet N., Hartmann L., 2004, ApJ, 617, 406

Neri L. J., Chavarria-K. C., de Lara E., 1993, A\&AS, 102, 201

Oliveira J. M., Foing B. H., van Loon J. T., Unruh Y. C., 2000, A\&A, 362, 615

Paunzen E., et al., 2002, A\&A, 392, 515

Pelletier G., Pudritz R. E., 1992, ApJ, 394, 117

Penston M. V., 1964, The Observatory, 84, 141

Pérez M. R., McCollum B., van den Ancker M. E., Joner M. D., 2008, A\&A, 486, 533

Poveda A., 1965, Boletin de los Observatorios Tonantzintla y Tacubaya, 4, 15

Rebull L. M., et al., 2002, AJ, 123, 1528

Reiter M., et al., 2018, ApJ, 852, 5

Robitaille T. P., 2011, A\&A, 536, A79

Robitaille T. P., 2017, A\&A, 600, A11

Romanova M. M., Owocki S. P., 2015, SSR, 191, 339

Romanova M. M., Ustyugova G. V., Koldoba A. V., Wick J. V., Lovelace R. V. E., 2003, ApJ, 595, 1009

Romanova M. M., Ustyugova G. V., Koldoba A. V., Lovelace R. V. E., 2004, ApJ, 610, 920

Rousselet-Perraut K., et al., 2010, A\&A, 516, L1

Ryabchikova T., Pakhomov Y., 2015, BaltA, 24, 453

Rydgren A. E., 1977, PASP, 89, 823

Rydgren A. E., Vrba F. J., 1987, PASP, 99, 482

Sagar R., Joshi U. C., 1983, MNRAS, 205, 747

Schneider F. R. N., Ohlmann S. T., Podsiadlowski P., Röpke F. K., Balbus S. A., Pakmor R., Springel V., 2019, Nature, 574, 211

Schöller M., et al., 2016, A\&A, 592, A50

Shu F., Najita J., Ostriker E., Wilkin F., Ruden S., Lizano S., 1994, ApJ, 429, 781

Sitko M. L., Simon T., Meade M. R., 1984, PASP, 96, 54

Strom S. E., Strom K. M., Brooke A. L., Bregman J., Yost J., 1972a, ApJ, 171,267

Strom S. E., Strom K. M., Yost J., Carrasco L., Grasdalen G., 1972b, ApJ, 173,353

Sung H., Bessell M. S., Chun M.-Y., Karimov R., Ibrahimov M., 2008, AJ, 135,441

Sung H., Stauffer J. R., Bessell M. S., 2009, AJ, 138, 1116

The P. S., de Winter D., Perez M. R., 1994, A\&AS, 104, 315

Thomas S. J., van der Bliek N. S., Rodgers B., Doppmann G., Bouvier J., 2007, in Hartkopf W. I., Harmanec P., Guinan E. F., eds, IAU Sympo- sium Vol. 240, Binary Stars as Critical Tools Tests in Contemporary Astrophysics. pp 250-253, doi:10.1017/S1743921307004103

Turner N. J., Benisty M., Dullemond C. P., Hirose S., 2014, ApJ, 780, 42

Valenti J. A., Piskunov N., 1996, A\&AS, 118, 595

Valenti J. A., Johns-Krull C. M., Linsky J. L., 2000, ApJS, 129, 399

Valenti J. A., Fallon A. A., Johns-Krull C. M., 2003, ApJS, 147, 305

Vasilevskis S., Sanders W. L., Balz A. G. A., 1965, AJ, 70, 797

Venuti L., et al., 2014, A\&A, 570, A82

Villebrun F., et al., 2019, A\&A, 622, A72

Vink J. S., Drew J. E., Harries T. J., Oudmaijer R. D., 2002, MNRAS, 337, 356

Vioque M., Oudmaijer R. D., Baines D., Mendigutía I., Pérez-Martínez R., 2018, A\&A, 620, A128

Wade G. A., Bagnulo S., Drouin D., Landstreet J. D., Monin D., 2007, MNRAS, 376, 1145

Walker M. F., 1956, ApJS, 2, 365

Warner J. W., Strom S. E., Strom K. M., 1977, ApJ, 213, 427

Wheelwright H. E., Oudmaijer R. D., Goodwin S. P., 2010, MNRAS, 401, 1199

Wichittanakom C., Oudmaijer R. D., Fairlamb J. R., Mendigutía I., Vioque M., Ababakr K. M., 2020, MNRAS, 493, 234

Wright E. L., et al., 2010, AJ, 140, 1868

Young A., 1978, PASP, 90, 144

Zwintz K., et al., 2009, A\&A, 502, 239

Zwintz K., Fossati L., Ryabchikova T., Kaiser A., Gruberbauer M., Barnes T. G., Baglin A., Chaintreuil S., 2013, A\&A, 550, A121 

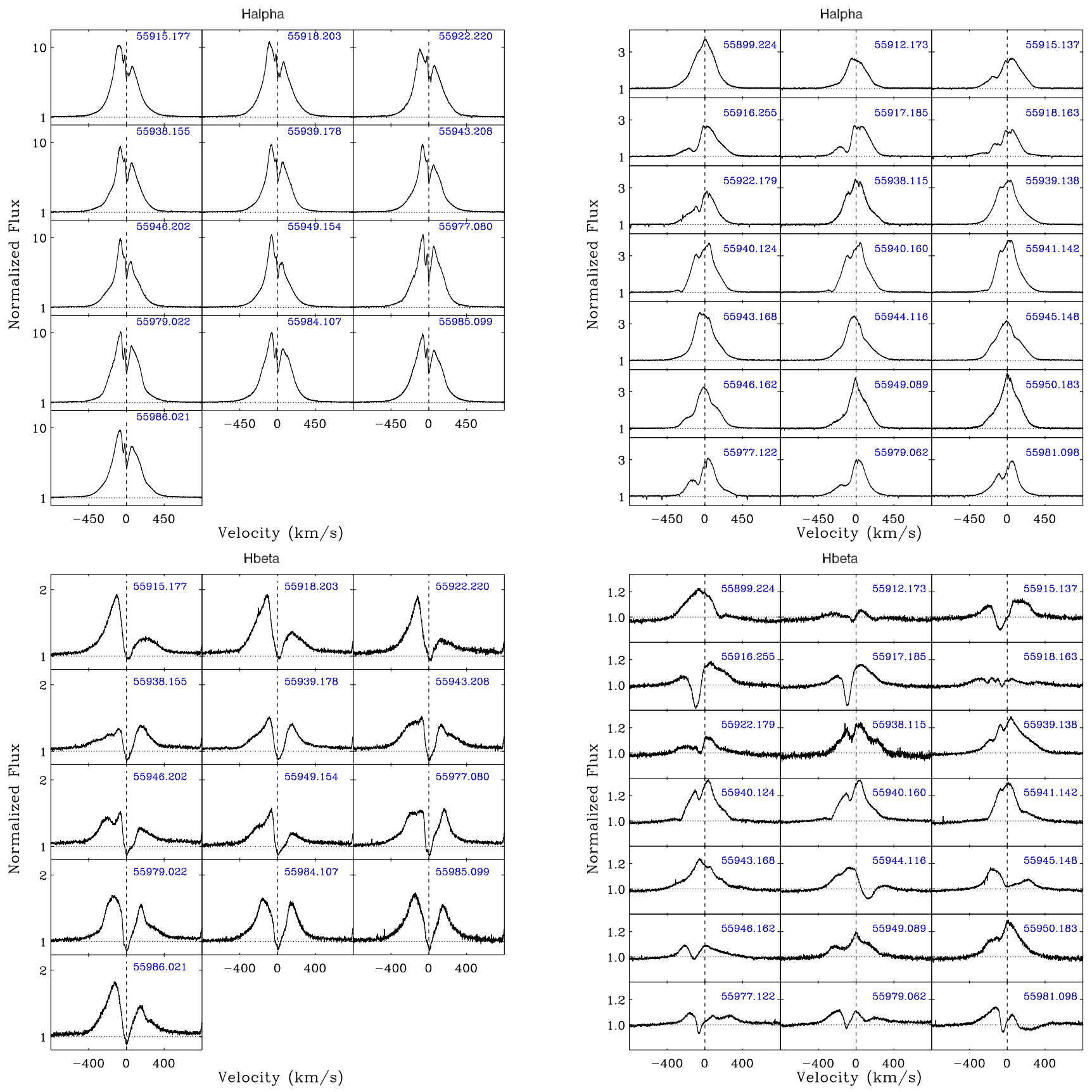

Figure A1. $\mathrm{H} \alpha$ and $\mathrm{H} \beta$ observed line profiles of V590 Mon. The Julian dates of the observations are shown in blue.

Figure A2. $\mathrm{H} \alpha$ and $\mathrm{H} \beta$ observed line profiles of HD 261941. The Julian dates of the observations are shown in blue.

This paper has been typeset from a $\mathrm{T}_{\mathrm{E}} \mathrm{X} / \mathrm{LT}_{\mathrm{E}} \mathrm{X}$ file prepared by the author.

\section{APPENDIX A: H $\alpha$ AND H $\beta$ OBSERVED PROFILES}

$\mathrm{H} \alpha$ and $\mathrm{H} \beta$ observed profiles of V590 Mon and HD 261941.

\section{APPENDIX B: H $\alpha$ THEORETICAL PROFILES CALCULATED WITH THE $C V$ MODEL}

Figures B1 and B2 show some examples of $\mathrm{H} \alpha$ theoretical profiles calculated with the $C V$ model (Lima et al. 2010), varying different system parameters. 


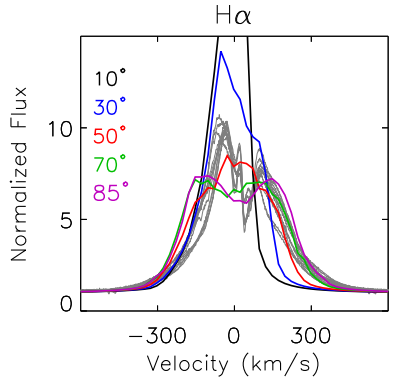

(a)

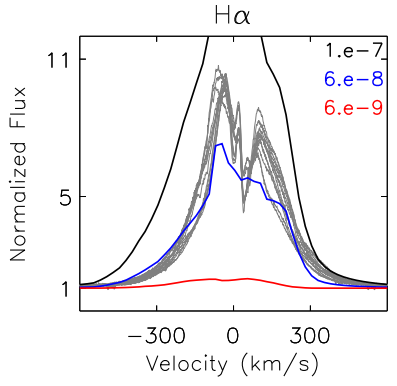

(b)
Figure B1. Theoretical line profiles of the $\mathrm{H} \alpha$ emission line computed with the $C V$ code with only the disc wind component, compared with the observed line profiles of V590 Mon (in grey). Each colored line corresponds to a model calculated wuth a different value of the varied parameter, keeping all the other parameters fixed at the values listed in Tab. B1. In panel (a) we varied the system inclination with respect to our line of sight (in degrees) and in (b) the mass loss rate (in $\mathrm{M}_{\odot} \mathrm{yr}^{-1}$ ).

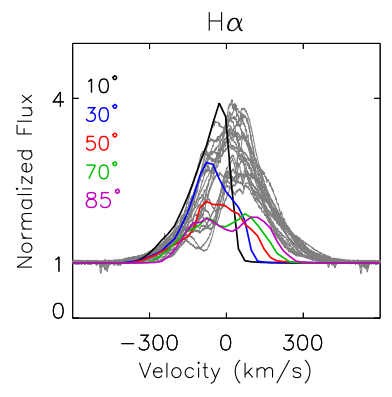

(a) Model 1

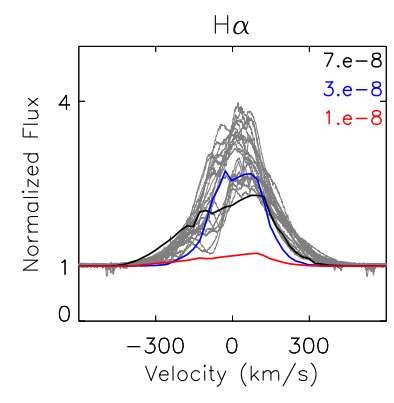

(b) Model 2
Figure B2. Theoretical line profiles of the $\mathrm{H} \alpha$ line computed with the $C V$ code with only the disc wind component, compared with the observed line profiles of HD 261941 (in grey). Each colored line corresponds to a model calculated with a different value of the varied parameter, keeping all the other parameters fixed at the values listed in Tab. B1. In panel (a) we varied the system inclination with respect to our line of sight (in degrees) and in (b) the mass loss rate (in $\mathrm{M}_{\odot} \mathrm{yr}^{-1}$ ).
Table B1. Set of parameters used in the $\mathrm{H} \alpha$ line models shown in Figs. B1 and $\mathrm{B} 2$.

Values

Fig. B1a Fig. B1b Fig. B1a Fig. B2b

\begin{tabular}{llcccc}
\hline \hline \multicolumn{1}{c}{ Parameters } & & & & & \\
\hline Stellar mass & $\mathrm{M}_{*}\left[\mathrm{M}_{\odot}\right]$ & 3.20 & 3.20 & 2.20 & 2.20 \\
Stellar radius & $\mathrm{R}_{*}\left[\mathrm{R}_{\odot}\right]$ & 2.15 & 2.15 & 2.90 & 2.90 \\
Photospheric temperature & $\mathrm{T}_{p h}[\mathrm{~K}]$ & 12500 & 12500 & 8500 & 8500 \\
Inclination of the system & $\mathrm{i}\left[^{\circ}\right]$ & $a$ & 51 & $a$ & 75 \\
Mass loss rate & $\dot{M}_{\text {loss }}\left[\mathrm{M}_{\odot} \mathrm{yr}^{-1}\right]$ & $4 . \mathrm{e}-8$ & $a$ & $3 . \mathrm{e}-8$ & $a$ \\
disc wind launching angle & $\vartheta_{0}\left[{ }^{\circ}\right]$ & 53 & 53 & 53 & 15 \\
Fiducial density & $\rho_{0}\left[\mathrm{~g} \mathrm{~cm}^{-3}\right]$ & $6 . \mathrm{e}-10$ & $b$ & $1.2 \mathrm{e}-10$ & $c$ \\
disc wind inner radius & $\mathrm{R}_{\text {di }}\left[\mathrm{R}_{*}\right]$ & 2.0 & 2.0 & 2.8 & 1.8 \\
disc wind outer radius & $\mathrm{R}_{\text {do }}\left[\mathrm{R}_{*}\right]$ & 10.0 & 10.0 & 10.0 & 10.0 \\
Maximum wind temperature & $\mathrm{T}_{\text {wind }}[\mathrm{K}]$ & 11200 & 11400 & 9600 & 9400 \\
\hline
\end{tabular}

$a$ Variable parameters. ${ }^{b}$ For model with $\dot{M}_{\text {loss }}=7 . \mathrm{e}-8 \mathrm{M}_{\odot} \mathrm{yr}^{-1}, \rho_{0}=1.7 \mathrm{e}-10 \mathrm{~g} \mathrm{~cm}^{-3}$. For model with $\dot{M}_{\text {loss }}=$ 3.e- $8 \mathrm{M}_{\odot} \mathrm{yr}^{-1}, \rho_{0}=7.8 \mathrm{e}-11 \mathrm{~g} \mathrm{~cm}^{-3}$. For model with $\dot{M}_{\text {loss }}=6 . \mathrm{e}-9 \mathrm{M}_{\odot} \mathrm{yr}^{-1}, \rho_{0}=6 . \mathrm{e}-11 \mathrm{~g} \mathrm{~cm}^{-3},{ }^{c}$ For model with $\dot{M}_{\text {loss }}=1 . \mathrm{e}-6 \mathrm{M}_{\odot} \mathrm{yr}^{-1}, \rho_{0}=9 . \mathrm{e}-10 \mathrm{~g} \mathrm{~cm}^{-3}$. For model with $\dot{M}_{\text {loss }}=1 . \mathrm{e}-8 \mathrm{M}_{\odot} \mathrm{yr}^{-1}, \rho_{0}=2.8 \mathrm{e}-11 \mathrm{~g} \mathrm{~cm}^{-3}$. For model with $\dot{M}_{\text {loss }}=6 . \mathrm{e}-7 \mathrm{M}_{\odot} \mathrm{yr}^{-1}, \rho_{0}=3.5 \mathrm{e}-10 \mathrm{~g} \mathrm{~cm}^{-3}$ 